\title{
The CLAS12 Forward Tagger
}

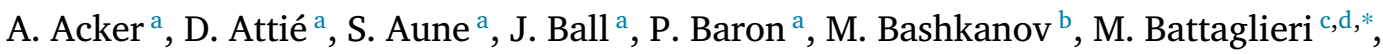

R. Behary ${ }^{\mathrm{e}}, \mathrm{F}$. Benmokhtar $^{\mathrm{e}}, \mathrm{A}$. Bersani ${ }^{\mathrm{c}}$, Q. Bertrand ${ }^{\mathrm{a}}$, D. Besin ${ }^{\mathrm{a}}, \mathrm{T}^{\mathrm{T}}$. Bey ${ }^{\mathrm{a}}$, P. Black ${ }^{\mathrm{f}}$,

P. Bonneau ${ }^{d}$, F. Bossù ${ }^{a}$, R. Boudouin ${ }^{a}$, M. Boyer ${ }^{a}$, P. Campero Rojas ${ }^{d}$, A. Casale ${ }^{c}$, A. Celentano ${ }^{c}$,

R. Cereseto ${ }^{\text {c }}$, A. Ciarma ${ }^{\text {g,h }}$, F. Cipro ${ }^{\text {c }}$, G. Charles ${ }^{\text {a }}$, G. Christiaens ${ }^{\text {a }}$, P. Contrepois ${ }^{\text {a }}$, M. Cook ${ }^{\text {d }}$,

A. D’Angelo ${ }^{g, h}$, R. De Vita ${ }^{c}$, M. Defurne ${ }^{\mathrm{a}}$, E. Delagnes ${ }^{\mathrm{a}}$, E. Fanchini ${ }^{\mathrm{c}}, \mathrm{S}$. Fegan ${ }^{\mathrm{b}}$, J. Fleming ${ }^{\mathrm{f}}$,

A. Filippi ${ }^{i}$, M. Garçon ${ }^{a}$, F. Georges ${ }^{a}$, K.L. Giovanetti ${ }^{j}$, D.I. Glazier ${ }^{k}$, R. Granelli ${ }^{a}$, N. Grouas ${ }^{a}$,

K. Hicks ${ }^{1}$, A. Hoebel ${ }^{d}$, S.M. Hughes ${ }^{f}$, C. Lahonde ${ }^{\mathrm{a}}$, L. Lanza ${ }^{\text {g,h }}$, M. Leffel ${ }^{\mathrm{d}}$, T. Lerch ${ }^{\mathrm{a}}$, T. Lemon ${ }^{\mathrm{d}}$,

K. Livingston ${ }^{\mathrm{k}}$, A. Manco ${ }^{\mathrm{c}}$, I. Mandjavidze ${ }^{\mathrm{a}}$, H.S. Mann ${ }^{\mathrm{j}}$, B. McKinnon ${ }^{\mathrm{k}}$, O. Meunier ${ }^{\mathrm{a}}$,

R. Miller ${ }^{\mathrm{d}}$, G. Miní c, Y. Mouden ${ }^{\mathrm{a}}$, P. Musico ${ }^{\mathrm{c}}$, M. Osipenko ${ }^{\mathrm{c}}, \mathrm{G}$. Ottonello ${ }^{\mathrm{c}}$, F. Parodi ${ }^{\mathrm{c}}$,

E. Pasyuk ${ }^{\mathrm{d}}$, P. Pollovio ${ }^{\mathrm{c}}$, F. Pratolongo ${ }^{\mathrm{c}}, \mathrm{S}$. Procureur ${ }^{\mathrm{a}}$, R. Puppo ${ }^{\mathrm{c}}$, C. Rossi $^{\mathrm{c}}$, M. Riallot ${ }^{\mathrm{a}}$,

M. Ripani ${ }^{\mathrm{c}}$, A. Rizzo ${ }^{\text {g,h }}$, F. Sabatié ${ }^{\mathrm{a}}$, C. Salgado ${ }^{\mathrm{m}}$, G.D. Smith ${ }^{\mathrm{f}}$, D. Sokhan ${ }^{\mathrm{k}}$, I. Stankovic ${ }^{\mathrm{f}}$,

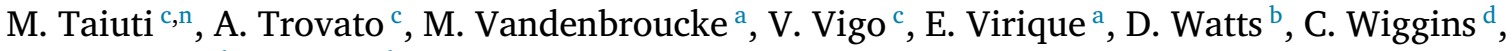

N. Zachariou ${ }^{\text {b }}$, L. Zana ${ }^{\text {d }}$

\footnotetext{
a IRFU, CEA, Université Paris-Saclay, F-91191 Gif-sur-Yvette, France

${ }^{\mathrm{b}}$ University of York, York YO10 5DD, United Kingdom

${ }^{c}$ INFN - Sezione di Genova, Via Dodecaneso 33, I-16146 Genova, Italy

d Thomas Jefferson National Accelerator Facility, Newport News, VA 23606, USA

${ }^{\mathrm{e}}$ Duquesne University, Pittsburgh, PA 15282, USA

${ }^{\mathrm{f}}$ University of Edinburgh, Edinburgh EH9 3FD, United Kingdom

${ }^{g}$ INFN, Sezione di Roma Tor Vergata, 00133 Rome, Italy

${ }^{\text {h }}$ Universitá di Roma Tor Vergata, 00133 Rome, Italy

${ }^{\mathrm{i}}$ INFN, Sezione di Torino, 10125 Torino, Italy

j James Madison University, Harrisonburg, VA 22807, USA

${ }^{\mathrm{k}}$ University of Glasgow, Glasgow G12 8QQ, United Kingdom

${ }^{1}$ Ohio University, Athens, $\mathrm{OH}$ 45701, USA

${ }^{\mathrm{m}}$ Norfolk State University, Norfolk, VA 23504, USA

${ }^{n}$ Universitá degli Studi di Genova, Via Dodecaneso 33, I-16146 Genova, Italy
}

\section{A R T I C L E I N F O}

\section{Keywords:}

Hadron spectroscopy

Low- $Q^{2}$ electron scattering

Electromagnetic calorimeter

$\mathrm{PbWO}_{4}$

APD

Hodoscope

Plastic scintillator

WLS fibers

SiPM

Gas tracking detector

MicroMegas

\begin{abstract}
A B S T R A C T
This document presents the technical layout and the performance of the CLAS12 Forward Tagger (FT). The FT, composed of an electromagnetic calorimeter based on $\mathrm{PbWO}_{4}$ crystals (FT-Cal), a scintillation hodoscope (FT-Hodo), and several layers of Micromegas trackers (FT-Trk), has been designed to detect electrons and photons scattered at polar angles from $2^{\circ}$ to $5^{\circ}$ and to meet the physics goals of the hadron spectroscopy program and other experiments running with the CLAS12 spectrometer in Hall $\mathrm{B}$.
\end{abstract}

\footnotetext{
* Corresponding author at: INFN - Sezione di Genova, Via Dodecaneso 33, I-16146 Genova, Italy. E-mail address: marco.battaglieri@ge.infn.it (M. Battaglieri).
} 


\section{Introduction}

An experimental program focused on the search for exotics and the study of rare mesons requires measurements of a broad range of final states in order to consolidate the possible evidence for their production by looking at different decay modes and exploring poorly studied reaction channels [1]. The characteristics of the detector and the trigger conditions foreseen for the experiment $-11 \mathrm{GeV}$ electron beam scattering on a 5-cm-long $\mathrm{LH}_{2}$ target with multiple particles in the final state - will allow measurements of many final states simultaneously. While the hadrons will be detected in the CLAS12 spectrometer [2], the electron scattered at very small angles $\left(2.5^{\circ}\right.$ to $4.5^{\circ}$ in polar angle) and low four-momentum transfer, $Q^{2}$, will be detected in the Forward Tagger (FT), i.e. in the kinematics of quasireal photoproduction. The FT specifications were thus defined to have optimal electron detection in this angular range, compatible with the high rate of electromagnetic background. To reconstruct the quasi-real photon variables, it is necessary to measure the scattered electron three momentum. The relevant quantities are:

- the energy $E_{e^{\prime}}$ : since the photon energy is given by $E_{\gamma}=v=$ $E_{\text {beam }}-E_{e^{\prime}}$ and its linear polarization by $P_{\gamma}=$ $\epsilon \sim\left(1+\frac{v^{2}}{2 E_{\text {beam }} E_{e^{\prime}}}\right)^{-1}$,

- the azimuthal angle $\phi_{e^{\prime}}$ to determine the polarization plane,

- the polar angle $\theta_{e^{\prime}}$ : since $Q^{2}=4 E_{\text {beam }} E_{e^{\prime}} \sin ^{2} \theta_{e^{\prime}} / 2$.

The FT is composed of an electromagnetic calorimeter (FT-Cal) to identify the electron in the energy range $0.5-4.5 \mathrm{GeV}$ by measuring its electromagnetic shower energy and to provide a fast trigger signal, a Micromegas tracker (FT-Trk) to measure the scattering angles $\left(\theta_{e^{\prime}}\right.$ and $\phi_{e^{\prime}}$ ), and a scintillation counter (FT-Hodo) to provide $e / \gamma$ separation. The FT-Cal and FT-Hodo also provide fast signals to trigger the data acquisition [3] in coincidence with signals from CLAS12. Fig. 1 shows a CAD rendering of the FT.

The calorimeter, the hodoscope, and the tracker are placed between the High Threshold Cherenkov Counter (HTCC) [4] and the torus magnet support [5], at about $185 \mathrm{~cm}$ downstream of the nominal target position. The close proximity to the beamline $\left(2.5^{\circ}\right.$ corresponds to $\sim 8 \mathrm{~cm}$ radial distance from the beamline) and the limited space available (at most $\sim 40 \mathrm{~cm}$ along the beam axis), requires a compact calorimeter of small radiation length and with very good radiation hardness. Fig. 2 shows a CAD drawing of the FT integrated in CLAS12. The FT-Hodo, placed in front of the calorimeter, is made of plastic scintillator tiles read-out by silicon photomultipliers via wavelength shifting fibers. The FT-Trk detector is located in front of the FT-Hodo to extend the acceptance of the FT down to $2.5^{\circ}$. All of these components were designed to fit within a $5.5^{\circ}$ Cone around the beam axis to have minimal impact on the operation and acceptance of the CLAS12 equipment in the forward direction.

\section{Detector layout}

\subsection{The calorimeter (FT-Cal)}

The FT-Cal has to fulfill demanding requirements in terms of: radiation hardness, light yield, shower containment (small radiation length and Moliere radius), scintillation decay time, and good energy and time resolution.

The electron energy resolution is a crucial factor to determine precisely the photon energy and to ensure the exclusivity of the measured reaction via the missing mass technique. However, since we are interested in low-energy electrons and high-energy photons, the energy resolution on the latter is significantly better than the resolution of the

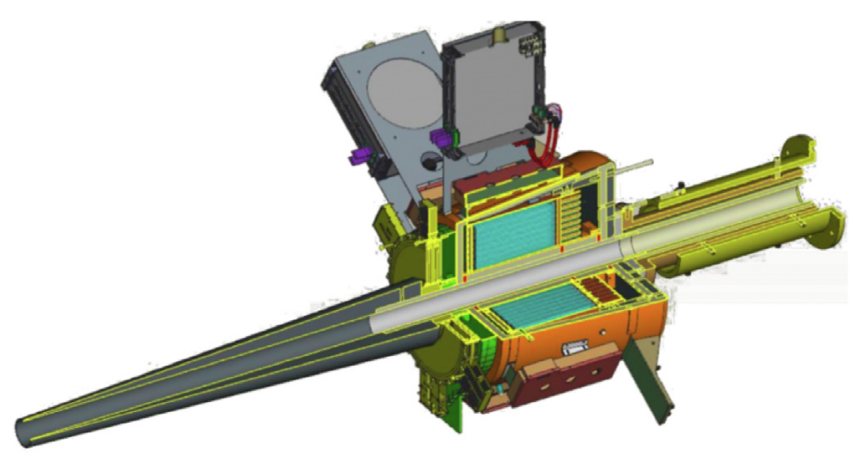

Fig. 1. CAD drawing of the Forward Tagger. The FT calorimeter shown in cyan is located at about $185 \mathrm{~cm}$ from the beam-target interaction point and is enclosed in a copper and Rohacell case to provide thermal insulation. The scintillation counter (green) and the tracker (yellow) are located in front of the calorimeter. A tungsten cone (gray) shields the FT from Møller electrons and other electromagnetic background (low-energy photons) created by the beam. The left side of this figure represents the upstream end of the detector. (For interpretation of the references to color in this figure legend, the reader is referred to the web version of this article.)

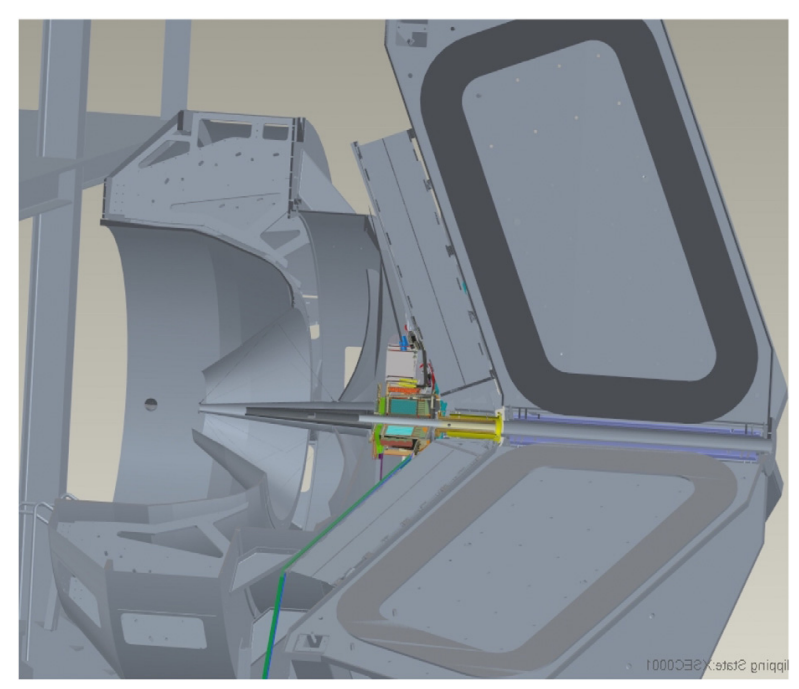

Fig. 2. CAD drawing showing the integration of the FT in CLAS12. The FT is located in the free space between the High Threshold Cherenkov Counter (HTCC) [4] and the first Drift Chamber (DC) region [6].

electron $^{1}$. The FT-Cal should have a fast scintillation decay time $(\tau \sim$ $10 \mathrm{~ns}$ ) to sustain high rates with small pile-up effects and to provide the scattered electron interaction time with good accuracy $(<1 \mathrm{~ns})$ in order to reject background and to identify the relevant signals via coincidence with CLAS12.

Due to the expected high rate from electromagnetic background $\left(\sim 120 \mathrm{MHz}\right.$ at the nominal luminosity of $10^{35} \mathrm{~cm}^{-2} \mathrm{~s}^{-1}$ ), the calorimeter should be highly segmented in the transverse direction. The size of each detection element should be comparable with the characteristic transverse size of the electromagnetic shower (Moliere radius) to contain the shower produced by incident electrons to a few readout cells, thus minimizing rates and pile-up. Finally, the photodetectors for the light read out should work in a sizable magnetic field and fit within the available space. Thus, standard photomultipliers (PMTs) cannot be used, while photodetectors based on semiconductors, e.g. avalanche photodiodes (APDs), have been shown to meet the required criteria.

\footnotetext{
1 For example, an electron energy resolution of $2 \%$ (at $1 \mathrm{GeV}$ ) would result in an energy resolution of $\sim 0.2 \%$ for the corresponding $10 \mathrm{GeV}$ photon, allowing the use of the missing mass technique for most of the reactions of interest.
} 


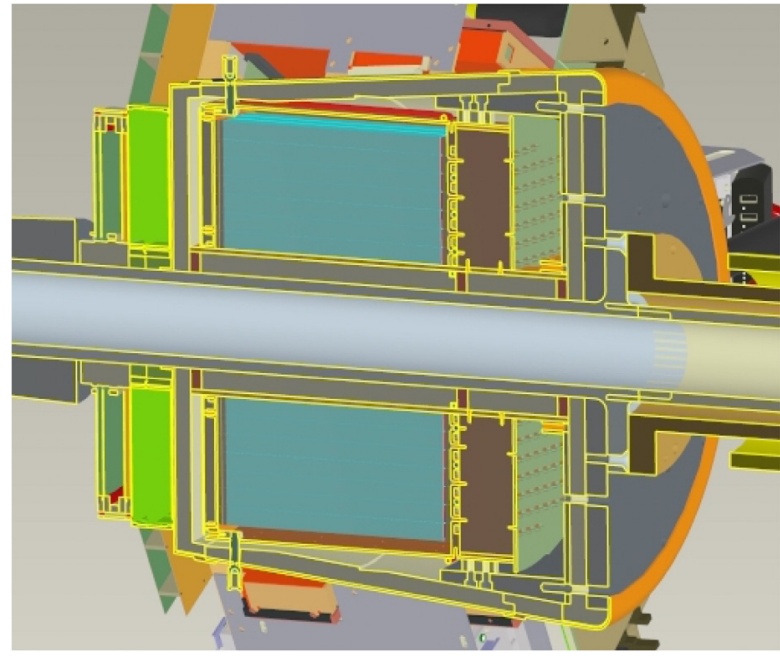

Fig. 3. CAD drawing of the FT-Cal showing a cross section of the detector. The crystals, in cyan, are enclosed in the copper thermal shield, in orange, surrounded by insulation, in light gray. On the downstream end of the crystals (right side of the figure), the preamplifiers motherboard is shown in green. The weight of the crystals is supported by the tungsten pipe, in dark gray, which is an integral part of the beamline.

To match the necessary requirements, lead tungstate $\left(\mathrm{PbWO}_{4}\right)$ was chosen as the scintillating material and Large-Area APDs (LAAPDs) as the readout sensors. A similar combination was used in the CMSECal [7], CLAS-IC [8], and PANDA-EMC [9] calorimeters. Lead tungstate has a fast scintillation decay time $(6.5 \mathrm{~ns})$, a small radiation length $(0.9 \mathrm{~cm})$, and small Moliere radius $(2.1 \mathrm{~cm})$. The drawback of limited light emission (about $0.3 \%$ of $\mathrm{NaI}(\mathrm{Tl})$ ) has been mitigated by using cooled $\mathrm{PbWO}_{4}$ Type-II crystals (same as used in the PANDA-EMC with better performance with respect to the $\mathrm{PbWO}_{4}$ Type I used in the CMS-ECal), matched to large-area photosensors to obtain a factor of four more light per MeV of deposited energy than the original CMS-ECal crystals.

With this design, based on GEANT simulations, an energy resolution on the order of $(2 \% / \sqrt{E(\mathrm{GeV})} \oplus 1 \%)$ is expected. Other crystals, such as LSO/LYSO or the very recent LaBr, share almost all of the good specifications of $\mathrm{PbWO}_{4}$ with a light yield more than 100 times larger. However, the higher costs and the limited experience in the manufacturing procedures excluded them from consideration as an alternative.

\subsubsection{Geometry and coverage}

The FT-Cal is made from $33215 \times 15 \times 200 \mathrm{~mm}^{3}$ parallelepiped $\mathrm{PbWO}_{4}$ Type-II crystals arranged around the beamline with full azimuthal angular coverage $\left(0^{\circ}<\phi<360^{\circ}\right)$ and small forward angle acceptance $\left(2^{\circ}<\theta<5^{\circ}\right)$. The crystals are placed with their long side parallel to the beamline to form a ring. Fig. 3 shows a CAD rendering of the calorimeter.

\subsection{2. $\mathrm{PbWO}_{4}$ crystals}

The FT-Cal $\mathrm{PbWO}_{4}$ Type-II crystals were produced by the Shanghai Institute of Ceramics, Chinese Academy (SICCAS) [10]. Since the light yield $(L Y)$ increases when lowering the temperature $T$ according to $d L Y / d T \sim 3 \% /{ }^{\circ} \mathrm{C}$, the calorimeter is stabilized in temperature and operated at $T \sim 0{ }^{\circ} \mathrm{C}^{2}$ Lower temperatures were not considered due to significant complications in the mechanical/thermal design, the reduced resistance to radiation, and the decay time degradation of the cooled $\mathrm{PbWO}_{4}$. The length of the crystals $(20 \mathrm{~cm}$ - corresponding to $\sim 22$ radiation lengths) was chosen to minimize the longitudinal loss and to match the available clearance.

\footnotetext{
2 At $T=0{ }^{\circ} \mathrm{C}$ the $L Y$ increases by a factor of two with respect to $T=25^{\circ} \mathrm{C}$.
}

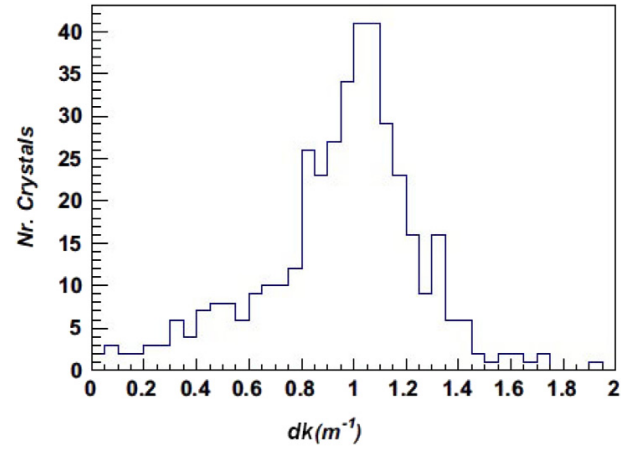

Fig. 4. Histogram of the radiation-induced absorption coefficient, $d k$, for all SICCAS FT-Cal $\mathrm{PbWO}_{4}$ crystals.

The $15 \mathrm{~mm} \times 15 \mathrm{~mm}$ size of the crystal front face provides a pixelization in the transverse plane of the $\mathrm{PbWO}_{4}$ crystals consistent with the Moliere radius. All crystals were characterized using the ACCOS (Automatic Crystal quality Control System) facility at CERN [11]. The geometrical dimensions, as well as the optical properties such as the longitudinal and transverse transmission and the relative light yield, were determined for each of the crystals. Samples that were outside of the required specifications were rejected and replaced by the manufacturer.

The absolute $L Y$ (number of detected photoelectrons per $\mathrm{MeV}$ deposited) was found to be $N_{p e}=220 \pm 20$ photoelectrons $/ \mathrm{MeV}$ at $T=0^{\circ} \mathrm{C} \pm 0.5^{\circ} \mathrm{C}$. For this measurement the crystal was wrapped on 5 of its faces with 3M Vikuiti reflective film and read out by a Hamamatsu S8664-1010 LAAPD operated at a gain $G=150$ connected with optical grease on the exposed face.

The scintillation decay time is also sensitive to the temperature. The time constant was measured using the Start-Stop or Delayed-Coincidence method at different temperatures. As expected, an increase in the decay constant was observed by decreasing the temperature. At $T=0^{\circ} \mathrm{C} \pm$ $0.5^{\circ} \mathrm{C}$, we found $\tau=13.5 \pm 0.6 \mathrm{~ns}\left(\tau_{2}=11.6 \pm 0.5 \mathrm{~ns}\right.$ and $\left.\tau_{1}=13.0 \pm 0.2 \mathrm{~ns}\right)$ when a single (double) exponential form was used to fit the data.

The radiation hardness of the crystals was measured by irradiating them with a dose of $30 \mathrm{~Gy}$ of low-energy photons using a ${ }^{60} \mathrm{Co}$ source at the Strahlenzentrum of Giessen University [12]. The longitudinal transmission was measured before and after the irradiation, calculating the variation as a function of the wavelength. The radiation hardness of the crystals was quantified by the radiation-induced absorption coefficient defined as:

$d k=\frac{1}{L} \frac{T_{b e f}}{T_{i r r}}$

where $T_{b e f}$ is the light transmission at $420 \mathrm{~nm}$, the peak of the $\mathrm{PbWO}_{4}$ emission spectrum, measured before irradiation, and $T_{i r}$ is the light transmission at the same wavelength after irradiation for crystals of a given length $L{ }^{3}$ Crystals exhibiting greater levels of radiation damage to light transmission have higher values of $d k$. All 332 crystals assembled in the FT-Cal were individually characterized: on average we found $T_{b e f}(420 \mathrm{~nm})=61.5 \pm 0.2(\sigma=3.2)$ and $T_{i r r}(420 \mathrm{~nm})=50.8 \pm 0.5$ ( $\sigma=4.9$ ). The resulting $d k$ distribution is shown in Fig. 4. These measurements were used to optimize the position of each crystal in the calorimeter, placing the crystals with the highest radiation resistance, and therefore lowest $d k$, in the areas where the highest radiation dose is expected.

\footnotetext{
${ }^{3}$ Crystal self-annealing was negligible since the two measurements were performed immediately before and after the short irradiation.
} 


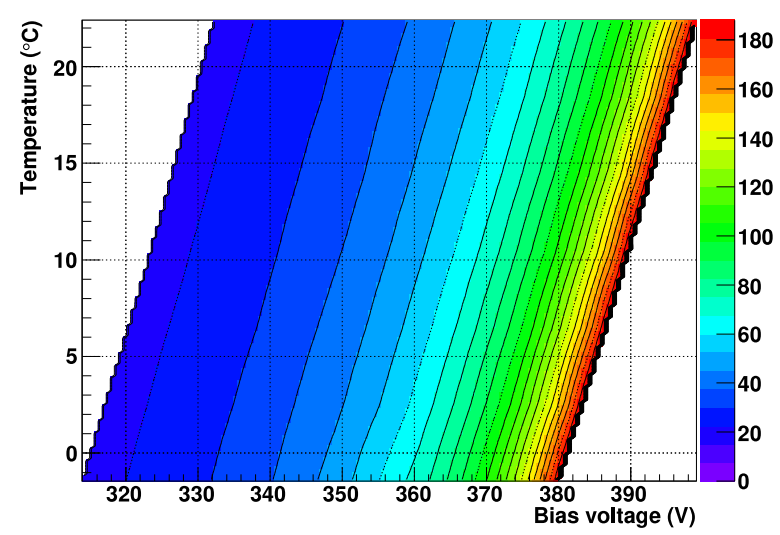

Fig. 5. Intrinsic gain of one representative APD as a function of the temperature and bias voltage.

\subsubsection{Light readout and electronics}

The FT-Cal uses $10 \times 10 \mathrm{~mm}^{2}$ (model Hamamatsu S8664-1010) LAAPDs to read out the $\mathrm{PbWO}_{4}$ scintillation light. APDs are only a few $\mathrm{mm}$ thick, have a large quantum efficiency at the $\mathrm{PbWO}_{4}$ light peak emission $(420 \mathrm{~nm})$, and are insensitive to magnetic fields. The main disadvantage is that, due to their low intrinsic gain $(\sim 50-200)$, the output signal is too small to be directly acquired, and needs to be amplified by a suitable circuit. APDs also need to be operated at a controlled temperature to avoid variations in gain and noise, but this does not represent a major complication since the crystals also are required to be stabilized in temperature. Each sensor used in the FTCal has been characterized by measuring its gain as a function of the applied bias voltage at a given temperature using an automated custom facility (see Ref. [13] for more details). The typical gain behavior $G\left(V_{\text {Bias }}, T\right)$ is shown in Fig. 5. The working point (bias voltage) was chosen in order to have the chosen gain $(G=150)$ in a reasonably stable region for small variations in the biasing. Silicon photomultiplier (SiPM) readout was not considered due to their limited dynamic range, which is not suitable for spectroscopic applications, and the limited experience (in term of reliability, radiation hardness, stability in time, etc.) with their use in large experiments at this time.

The APD current signal is converted to a voltage pulse that is transmitted to the subsequent electronics chain via a transimpedance amplifier (i.e. an amplifier that converts an input current pulse into an output voltage pulse, without performing any time integration). This amplifier has been developed in collaboration with the Service Electronique pour la Physique (SEP) of the Institut de Physique Nucléaire (IPN) in Orsay. The amplifier $\mathrm{ENC}^{4}$ was measured at the operating temperature of $T=0{ }^{\circ} \mathrm{C}$, with ENC $10400 e^{-}$(RMS) for a nominal gain of $G=600$. This corresponds to about $3 \mathrm{MeV}$ (RMS) on the measured energy. The amplified signal is read out using the custom JLab flash ADC VME board (a 16-channel, 12-bit, 250-MHz digitizer; referred to as the FADC250). The measurement of the full waveform allows for the derivation of both the charge and time of the hit with the required accuracy.

\subsubsection{Light monitoring system}

Lead tungstate scintillating crystals are known as an appropriate material for use in total absorption shower detectors. Unfortunately, although relatively radiation tolerant, their light output is reduced when exposed to electromagnetic radiation and recovers when the radiation source is removed. Further complications arise because at the same irradiation intensity, changes in light output may vary from one

4 The ENC, equivalent noise charge, is defined as the charge transported by an input signal giving, at the output of the amplifier, a signal whose amplitude is equal to the RMS of the output noise.

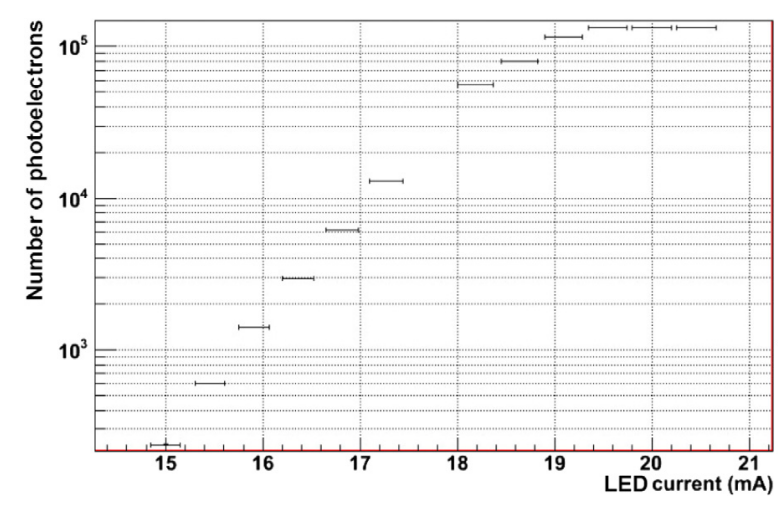

Fig. 6. Number of photoelectrons as a function of the LED driver current. The corresponding energy per crystal ranges from $10 \mathrm{MeV}$ to $10 \mathrm{GeV}$.

crystal to another. In order to maintain the intrinsic energy resolution, the crystals have to be continuously monitored and, if necessary, recalibrated by changing the supply voltage. The monitoring system should be able to test the response over time of the whole chain: crystal, APD, readout electronics. Among the different possible options (radioactive source, laser, and LED) we used an LED-based Light Monitoring System (LMS). In spite of the need for thermal control, LEDs offer the considerable advantage that the matching with crystals is simpler than for lasers, since each crystal can have an LED in front of it and the arrangement of power lines and electrical connections is less critical than for optical fibers. The main disadvantage is related to the complexity of the electronic circuitry. To cover a large light intensity range while maintaining good timing performance, each LED needs a separate driver, which leads for a calorimeter of significant size, to a large number of electronic circuits.

With LEDs it is possible to obtain a shape and a duration of the monitoring-light flash that is similar to the features of the crystal scintillation light. In fact, the emission spectrum of the monitoring light can be chosen to be similar to the radio-luminescence spectrum of $\mathrm{PbWO}_{4}$, the effective optical path length for monitoring light in the crystal can be matched to the average path length of the scintillation light produced by an electromagnetic shower, and the pulse length can be tuned to reproduce the $\mathrm{PbWO}_{4}$ scintillation decay time. We chose a blue light LED with wavelength close to the $430 \mathrm{~nm}$ emission peak of the $\mathrm{PbWO}_{4}$ crystal, where radiation damage may have the maximum effect.

Each crystal is equipped with a separate LED, located on its upstream face, at the opposite end with respect to the light sensors and electronics. The intensity can be varied in the range from 500 to 100,000 photons, pulsed at a variable rate from $62 \mathrm{~Hz}$ to $8 \mathrm{kHz}$, with a pulse rise time of $\sim 1 \mathrm{~ns}$ and a time jitter of less than $200 \mathrm{ps}$. The system has been designed to work in the temperature range from $-25{ }^{\circ} \mathrm{C}$ to $+30{ }^{\circ} \mathrm{C}$. The LEDs placed in the closed environment of the crystal are kept at constant temperature with an accuracy of $\Delta T=0.1^{\circ} \mathrm{C}$. The LED monitoring system is split in two boards: one containing the control logic and the LED driver circuits, and the other, mounted in front of the FT-Cal crystals, hosting the LEDs. The two boards are connected via a board-to-board connector that allows the required flexibility to match the FT-Cal geometry and positioning. The LED drivers are controlled by an on-board PIC32 micro-controller accessible remotely via Ethernet. Each LED is individually set by a programmable length and intensity pulse. The system is triggered by an internal clock or by an external signal. In both cases the trigger signal is available for a precise time reference.

The performance of the LED driver has been measured by coupling a single monitoring channel to a PMT. The performance of the system is reported in Figs. 6 and 7, where the measured number of photoelectrons as a function of the LED current and the measured time resolution 


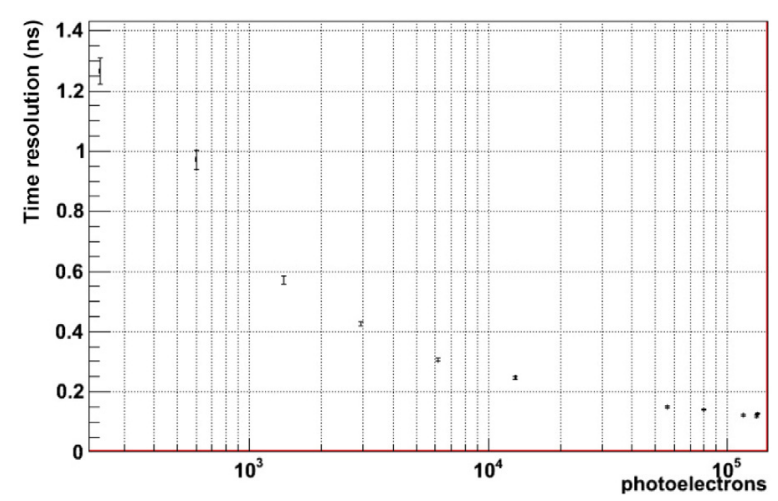

Fig. 7. Time resolution (measured as the time difference of the trigger signal and the PMT pulse) as a function of the LED light intensity.

as a function of the number of photoelectrons are shown. ${ }^{5}$ Rescaling the results to take into account the APD readout and the crystal $L Y / \mathrm{MeV}$, the equivalent energy ranges from $10 \mathrm{MeV}$ ( 500 photoelectrons - phe) to $10 \mathrm{GeV}$ (500k phe) perfectly match to the expected energy collected by each crystal. A time resolution of 100 ps is reached at high light intensity. The long-term stability of the system has been measured over a 100-hr run at $T=+18{ }^{\circ} \mathrm{C}$. The stability of each individual channel was found to be in the range of $2 \%$; when the ratio of any two channels is considered, the stability is at a level of a few parts per thousand.

\subsubsection{Slow controls and interlocks}

The FT-Cal slow controls are part of the CLAS12 EPICS system [3]. The APDs need to be reverse-biased with a positive high-voltage power source. The APD intrinsic gain depends on the bias voltage with $\frac{1}{G} \frac{\Delta G}{\Delta V} \sim$ $4 \%$ and, therefore, the power supply needs to be stable in time, with low output noise. We chose the CAEN A1520P board designed for the CMS electromagnetic calorimeter. The power supply fulfills all of our requirements in terms of dynamic range, linearity, and noise. Each board is equipped with 12 independent channels that each control a group of 10 APDs with relative gain variations not greater than $3 \%$.

The amplifiers used in the FT-Cal need to be operated with $+5 \mathrm{~V}$ and $-5 \mathrm{~V}$. The power consumption from each of the two voltage sources is approximately $70 \mathrm{~mW}$, almost independent of the event rate, giving a power consumption of $\sim 140 \mathrm{~mW}$ per board, for a total of $56 \mathrm{~W}$ for a 400-channel calorimeter. The full FT-Cal is powered by a Wiener MPOD MPV8008L power supply. Sensing feedback is implemented to compensate the voltage drop across the connecting cables.

Temperature regulation is provided by a Lauda XT150 chiller unit. This is a self-regulating unit and does not require external feedback, however, the settings and monitored parameters are sent to EPICS for recording via a streamDevice module. The FT-Cal temperature is monitored by a set of PT100 thermoresistors located at different positions within the crystal assembly and read by a cRio module, which is part of the interlock system. The flow of nitrogen gas, which is purged in the preamplifier area to prevent moisture build-up at low temperature, is measured with a flowmeter and monitored by the same cRio system. The latter is also used to read the output of two humidity sensors located in the preamplifier area.

The $c$ Rio system is the main component of the interlock system that was designed to provide a fast shutdown mechanism for all critical components in case abnormal conditions are detected. The parameters that are monitored are the FT-Cal temperatures, the nitrogen flow, and the humidity. If any of the measured values is found to be outside userdefined ranges, the system disables the FT-Cal high voltage (HV) and low voltage (LV) crates and stops the chiller to prevent any damage to the detector or surrounding elements.

5 The time resolution is defined as the width $(\sigma)$ of the time difference distribution between the trigger signal and the PMT output.

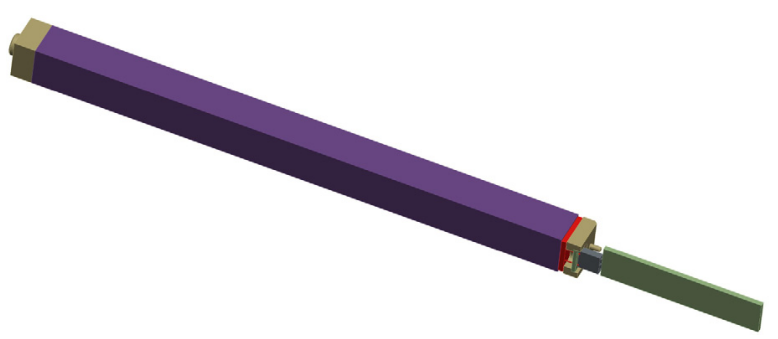

Fig. 8. Single crystal assembly: from the left (front) to the right (back), the PEEK support that holds the nose with the LED housing, the crystal wrapped in 3M Vikuiti reflective film, the LAAPD in the PEEK housing, and the preamplifier.

\subsubsection{Mechanical design}

The mechanical design of the calorimeter is driven by three considerations: minimization of the empty spaces between the crystals, cooling to $0{ }^{\circ} \mathrm{C}$, and optimal coverage of the required acceptance without interference with the rest of CLAS12.

The building blocks of the calorimeter are the individual leadtungstate crystals. Each crystal is $15 \times 15 \times 200 \mathrm{~mm}^{3}$, for a weight of $370 \mathrm{~g}$. Each crystal is optically coupled to an LAAPD on its back face and to an LMS LED on its front face for calibration. To achieve the maximum light collection efficiency, the APD covers almost the entire area of the downstream end of the crystal, so the LED for monitoring has to be mounted on the upstream end. This reflects onto the mechanical design of the single-crystal assembly as a monolithic, self-supporting element made of the crystal itself, the APD, the reflective wrapping, and the crystal support structure. To avoid dead volume in the detector, the mechanical support for each crystal is provided only by the wrapping. We chose 3M Vikuiti reflective film. This material is non-conductive, has a reflectivity higher than aluminized Mylar and, if properly heatformed, can keep together the different parts of the assembly. The reflective film is glued on the sides of a pair of front/back PEEK custommachined blocks that hold the LAAPD and the LED, respectively. Fig. 8 shows a CAD rendering of the single crystal assembly from the front PEEK support to the preamplifier.

The crystal assemblies are installed in a matrix to provide complete shower containment for electrons in the FT-Cal angular acceptance. Two copper plates, placed in front of and on the back of the crystals, define the positioning for the crystal assemblies. On the APD side, the preamplifiers, one for each crystal, are connected to the readout motherboard, which is designed to provide power distribution and signal collection for each channel. The mechanical structure allows for the replacement of individual preamplifiers if needed. The front and back copper plates are connected by a copper cylinder on the outside and by an inner copper shield to form a closed vessel that surrounds the crystal matrix to provide proper grounding and the required thermal stability and uniformity. Cooling is provided by $5-\mathrm{mm}$ diameter copper pipes installed on the outside of the vessel as shown in Fig. 9.

The FT calorimeter was designed to operate between $0{ }^{\circ} \mathrm{C}$ and room temperature. The FT-Cal cooling is achieved via circulation of coolant in the circuit attached to the rear copper plate and on the inner and outer copper vessels. The cooling system was designed to compensate the heat load in the region surrounding the FT, taking into account $20 \mathrm{~mm}$ of insulating foam (polyisocianurate thermal conductivity $0.024 \mathrm{~W} / \mathrm{mK}$ ) and from the amplifiers, which dissipate $\sim 50 \mathrm{~W}$. The insulation is less effective between the calorimeter and the inner tungsten pipe that holds the entire FT (see Section 3) because of the limited space for the insulation and the presence of the support structures that bring the overall thermal conductance in that region to $0.056 \mathrm{~W} / \mathrm{mK}$.

During the design phase, Finite Element Analysis calculations were performed to optimize the cooling circuit and the insulation parameters in order to reach the design temperature and uniformity. These studies 


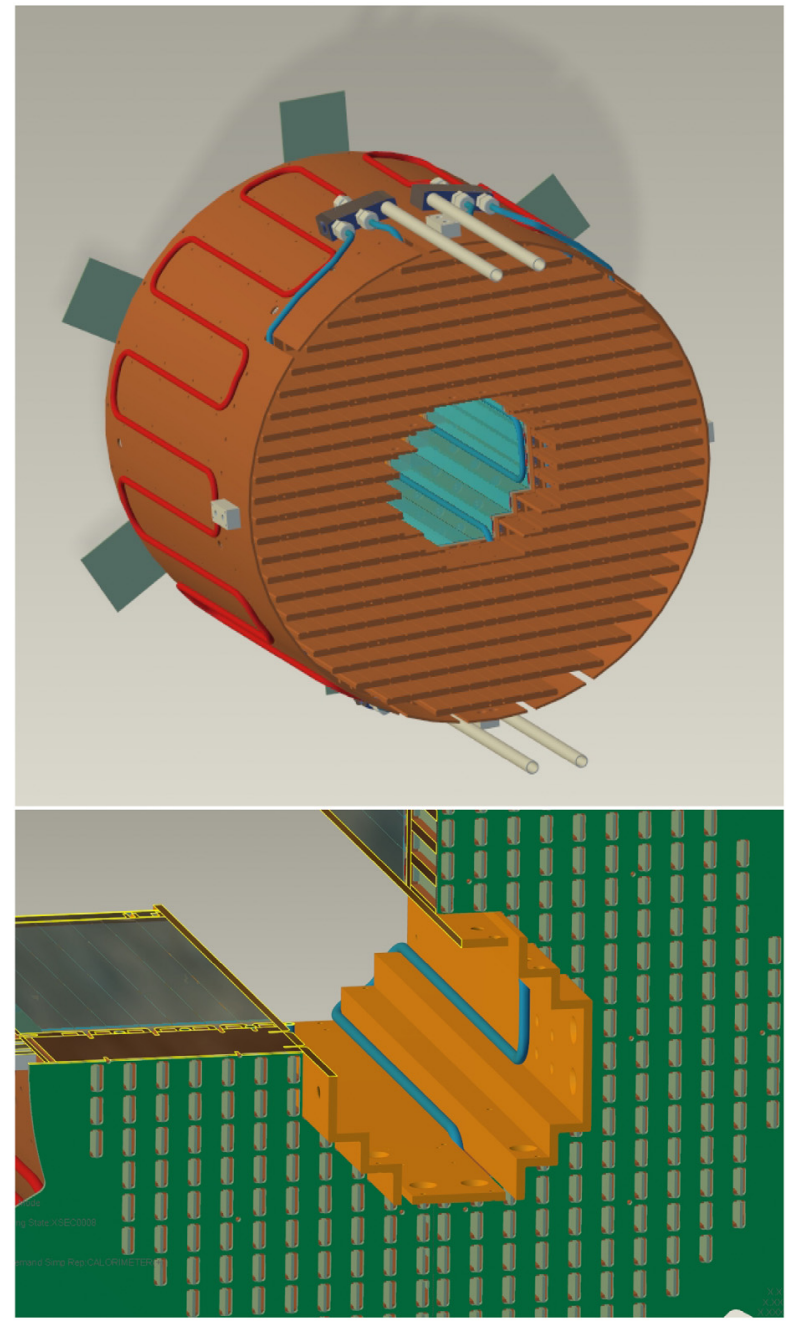

Fig. 9. The copper thermal/grounding shield for the FT-Cal. The top figure shows the ensemble of the copper shield with the cooling pipes shown in red and blue. These are located on the back plate, on the outer cylinder, and on the inner shield. The bottom figure shows the cooling pipe circuit inside the inner shield. (For interpretation of the references to color in this figure legend, the reader is referred to the web version of this article.)

indicated that the coldest part of the external calorimeter enclosure is the tungsten cone, which is expected to stabilize at a temperature just above the dew point. Measurements performed after the calorimeter assembly confirmed these results.

\subsection{The hodoscope (FT-Hodo)}

The primary aim of the FT-Hodo is to discriminate between photons and electrons that produce an electromagnetic shower in the calorimeter. Specifically, electrons are identified by hits in the hodoscope array that are correlated in both position and time with a cluster observed in the calorimeter. The FT-Hodo is comprised by an array of 232 plastic scintillator (Eljen-204) tiles segmented in two layers to suppress contributions from the splash-back of the electromagnetic shower created by events depositing energy in the FT-Cal. The scintillators provide fast timing and sufficient resistance to radiation damage for use in the high-rate and high-dose environment of the FT. The geometry and readout of the hodoscope are constrained by the surrounding apparatus. Specifically, the device is positioned upstream of the FT-Cal, fitting into a circular disk of diameter $330 \mathrm{~mm}$ and $42 \mathrm{~mm}$ depth. The readout is achieved using $3 \times 3 \mathrm{~mm}^{2}$ Hamamatsu S13360-3075PE SiPMs $(50 \%$

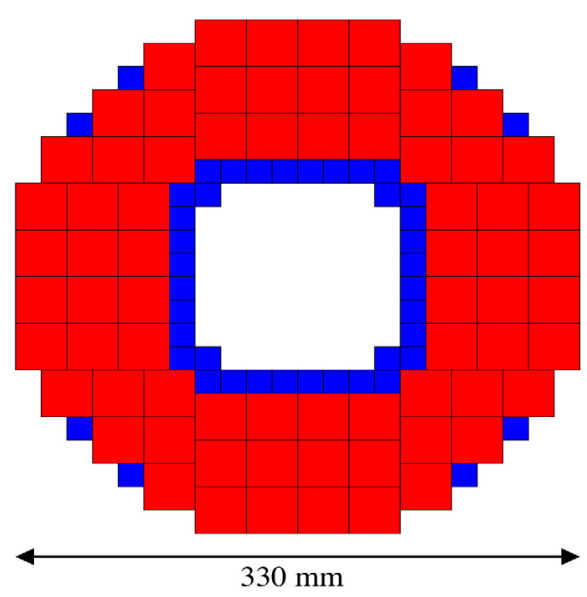

Fig. 10. The arrangement of plastic scintillator tiles in the FT-Hodo. The blue (red) squares represent the $15 \mathrm{~mm} \times 15 \mathrm{~mm}(30 \mathrm{~mm} \times 30 \mathrm{~mm})$ tiles for each layer. (For interpretation of the references to color in this figure legend, the reader is referred to the web version of this article.)

photon detection efficiency for $450 \mathrm{~nm}$ photons) coupled to 5-m-long clear optical fibers (Kuraray clear-PSM with attenuation length $>10 \mathrm{~m}$ ), which are fusion spliced to $\sim 30$-cm-long wavelength shifting (WLS) Kuraray Y11 fibers (attenuation length of $>3.5 \mathrm{~m}$ ), embedded in the scintillator tiles. The splicing induces a photon loss of less than $2 \%$, where the use of optical fibers allows the captured light to be transported with a light loss of less than $\sim 40 \%$ over the 5 -m path to the SiPM. This readout design of the FT-Hodo addresses the need to minimize material in the detector acceptance, to operate in regions of high magnetic fields produced by the CLAS12 solenoid and torus magnets, and to tolerate the high-background radiation environment.

Each layer of the FT-Hodo is comprised of $4415 \mathrm{~mm} \times 15 \mathrm{~mm}$ (P15) and $7230 \mathrm{~mm} \times 30 \mathrm{~mm}$ (P30) scintillators arranged as shown in Fig. 10. The upstream and downstream layers utilize 7-mm and 15-mm-thick scintillator tiles, respectively. The upstream (thin) layer is employed to reduce photon conversion in the FT-Hodo, while the thicker layer provides the signal with the most accurate timing information for the event. To increase the number of scintillation photons collected from each tile, four WLS fibers were embedded in the P30 tiles and 2 in the P15 tiles. In addition, the WLS fibers were glued with Epotek 301-2 glue inside diagonal holes to maximize the path length in the scintillator and to allow for the tiles to be arranged without any dead space between the elements.

Each tile was polished and painted with two layers of Bicron BC620 reflective paint for the sides and 3 layers for the scintillator faces and secured in position on the surface of a 1-mm-thick plastic support board. There is a 9-mm clearance for each layer for routing the optical fibers to the readout electronics through a $\Delta$-shaped sheathing on the bottom end of the FT-Hodo. The front and back faces are covered by light-proof carbon fiber material that is screwed onto supporting structures made out of hexagonal plastic spacers (15-mm wide and 22or $15-\mathrm{mm}$ tall depending on the layer). This results in a total detector thickness of $42 \mathrm{~mm}$. A 1-mm-thick plastic strip traces the outer contour of the FT-Hodo and is glued onto the spacer supports. Fig. 11 shows a CAD drawing of the FT-Hodo highlighting one layer of tiles, the location of the plastic supports for the light-proofing structure, and the plastic strip.

With the typical maximum radiation doses determined through Geant4 simulations with realistic beam and target parameters, and without the shielding effects of the Møller cone (see Section 3), the FT-Hodo will experience a light loss of $20 \%$ in the WLS fibers after 3.5 years, whereas the plastic scintillators will experience a light loss of $20 \%$ after 300 years [14]. Both scintillators and fibers also show 


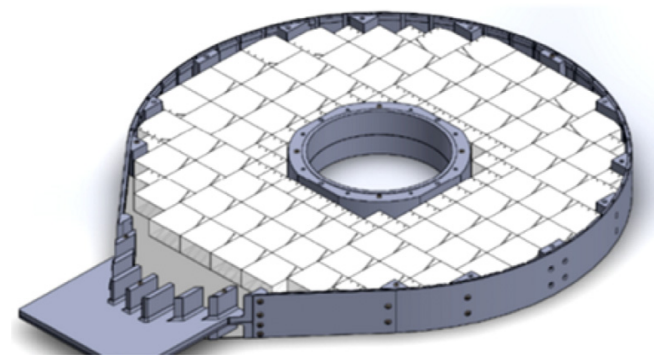

Fig. 11. CAD drawing of the FT-Hodo showing one layer of tiles, the locations of the plastic spacers, and the plastic strip that traces the outer contour.

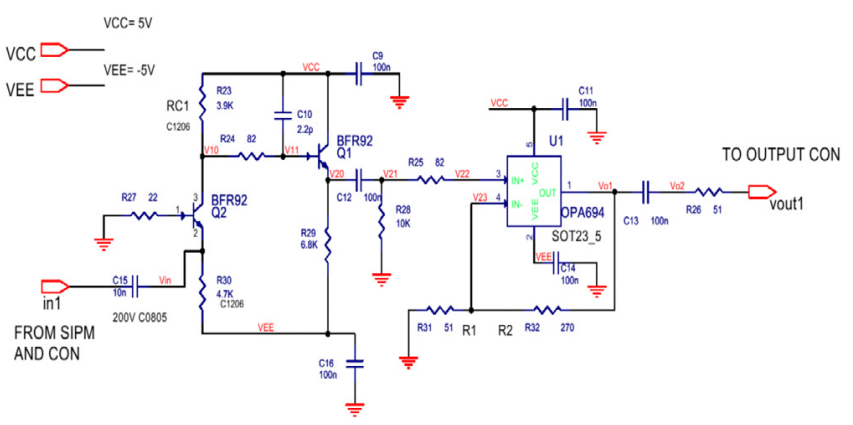

Fig. 12. Schematic of a single channel of the amplifier board for the SiPM.

natural annealing processes, which can effectively compensate for the radiation damage [14].

The analog signal from the SiPM is fed directly to a custom-designed preamplifier board designed by the INFN-Genova Electronics Group. The boards host 8 independent channels, each coupled to a SiPM and are mounted in pairs in the slots of a custom crate, mechanically compatible with the VME standard. The 16 SiPMs connected to each pair of boards are mounted on a mezzanine printed circuit board, which distributes the bias HV to each SiPM and collects their signals for the amplifier inputs. The schematic of one channel of the SiPM amplifier board, excluding the HV bias network is shown in Fig. 12. The first stage is based on a bipolar junction NPN transistor in a common base configuration, while the second is composed of an OPA694 operational amplifier in a non-inverting configuration. The two BRF92 transistors have been chosen since they are low-noise transistors with a high cutoff frequency and good stability. The two stages are coupled together with a $100 \mathrm{nF}$ capacitor to remove the DC component of the signal from the second transistor. The amplifier is coupled to the output connector through a $100 \mathrm{nF}$ capacitor and a $50 \Omega$ resistor to remove any DC component from the last stage, and to match the impedance of the output cable.

The signal from each SiPM after amplification is continuously digitized by the JLab FADC250 boards and, if the trigger condition is satisfied, samples are stored for further analysis. The data acquisition and slow controls system for the FT-Hodo are similar to the FT-Cal (see Section 2.1.3 for more details). The SiPMs operate with a bias voltage of $50-55.5 \mathrm{~V}$, which is provided by three CAEN A1737P HV boards. 30 independent HV channels are used to operate each SiPM board that host 8 sensors. These groups of $8 \mathrm{SiPMs}$ were selected according to their gain. The HV distribution to the groups of 8 SiPMs is implemented on the mezzanine boards that also hosts a compensation circuit to allow for the independent regulation of each SiPM bias voltage up to a maximum of $0.4 \mathrm{~V}$. The low voltage system used for the FT-Hodo is the same as the one used for FT-Cal. Controls of both the HV and LV for the detector are provided by the CLAS12 EPICS slow controls system [3]. Similarly to the FT-Cal, the status of the critical components, in this case the temperature of the preamplifier crate, is incorporated into the interlock

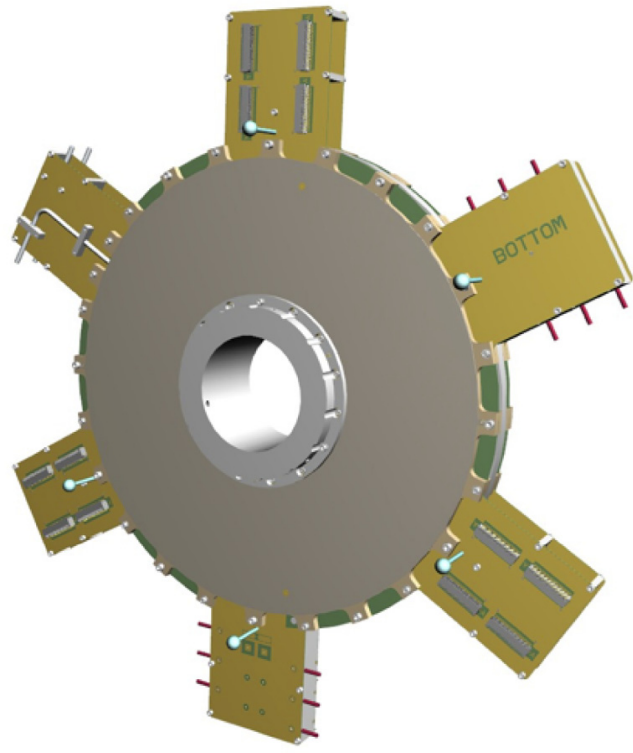

Fig. 13. 3D view of the upstream face of the FT-Trk Micromegas tracker equipped with front-end electronics.

system that is programmed to disable the HV and LV crates if abnormal conditions are detected.

\subsection{The micromegas tracker (FT-Trk)}

For a precise determination of the scattered electron angle, a tracker complements the FT-Cal and FT-Hodo detectors. The FT-Trk uses the same technology adopted by the CLAS12 central and forward Micromegas detectors. We refer to Ref. [15] for a detailed description of these devices. In this section we describe the specific design of the FT-Trk.

Two double-layers of Micromegas detectors are located in front of the hodoscope, in the space between the FT and the HTCC [4]. The two detectors are indeed a good compromise to achieve an efficient background rejection and track reconstruction with a low material budget. Each layer is composed of a double-faced Micromegas disk built on a common printed circuit board (PCB). Each side of the PCB displays strips, the downstream strips being perpendicularly oriented to the upstream strips. This particular geometry enables the determination of the $(x, y)$ coordinates (perpendicular to the beam $z$-axis) of a track. To limit the number of electronics channels, the pitch chosen was $500 \mu \mathrm{m}$, which leads to a resolution better than $500 / \sqrt{12} \sim 150 \mu \mathrm{m}$. A drift space of $5 \mathrm{~mm}$, together with an amplification gap of $128 \mu \mathrm{m}$, provides good efficiency. The two double-layers, centered on the beam axis, cover polar angles from $2.5^{\circ}$ to $4.5^{\circ}$ with an active area defined between a $70 \mathrm{~mm}$ inner radius and a $143 \mathrm{~mm}$ outer radius. The total number of channels is 3072 . Fig. 13 shows the CAD implementation of the detector. The FT-Trk readout uses the same data acquisition scheme adopted for the CLAS12 Barrel Micromegas Tracker (BMT) [15], which consists of a Front-End Unit (FEU) and a Back-End Unit (BEU).

The front-end electronics are responsible for signal preamplification, shaping, buffering during the trigger generation process, data digitization, and compression. Due to the limited space available, the front-end electronics are designed to be placed off-detector. Microcoaxial cable assemblies connect the detectors and the front-end boards. The non-amplified analog signals transit via the cable assemblies from the chambers to the front-end electronics. The 512-channel FEUs are housed in $4 \mathrm{U}$ crates attached to the FT-Cal mechanical supports, which are located in the geometrical shadow of the CLAS12 torus coils. The back-end electronics are responsible for data concentration, providing 
the interface to the CLAS12 event building system and are the same units used for the BMT [15].

Each Micromegas layer is powered with $450 \mathrm{~V}$ for the micro-mesh and $1000 \mathrm{~V}$ for the drift electrode. The FT-Trk front-end power supply is located $12 \mathrm{~m}$ away from the crates. The $15 \mathrm{~W}$ power produced by each crate is dissipated by compressed air. An interlock system between the cooling infrastructure and the low voltage power supply prevents powering the front-end crates when cooling is off.

The gas used is a mixture of argon, isobutane (up to 10\%), and $\mathrm{CF}_{4}$ (up to 5\%). The use of $\mathrm{CF}_{4}$ ensures good time resolution (around 10-15 ns). The gas distribution system is the same one used by the BMT.

\section{Integration in CLAS12}

The FT mechanical design was driven by the geometrical constraints imposed by the other CLAS12 sub-detectors, geometrical acceptance optimization, and performance optimization, taking into account the cooling requirements, material budget, and front-end electronics location. The FT detects electrons scattered between $2.5^{\circ}$ and $4.5^{\circ}$ with respect to the beam axis. To provide this acceptance, the FT calorimeter must cover down to $2^{\circ}$ and up to $5^{\circ}$ with lead tungstate crystals to have a good containment of electromagnetic showers at the edges of the polar angular range. Since no massive materials are allowed at angles larger than $5.5^{\circ}$, the crystals, cooling system, mechanical supports, and tungsten shielding have been optimized in a very compact design. Outside of $5.5^{\circ}$ the only materials are very low-density $\left(35 \mathrm{~kg} / \mathrm{m}^{3}\right)$ insulation and routing for cabling and services in the geometrical shadow of the CLAS12 detector where the torus magnet coils are located.

The FT is built from several components that can be grouped as follows:

- the inner tungsten pipe,

- the tungsten cone acting as a Møller electron shield,

- the FT-Trk tracker,

- the FT-Hodo hodoscope,

- the FT-Cal calorimeter,

- the front-end electronics,

- cabling and services.

From the mechanical point of view, the most challenging aspect is the integration of the calorimeter, due to the weight and fragility of the crystals, and the relative positioning and alignment of the FT components.

\subsection{Constraints from other sub-detectors}

The FT must be centered on the beamline between the HTCC and the first set of the DCs [6]. The HTCC can be retracted in the upstream direction to give access to the FT. In its operating position, the HTCC extends to $1730 \mathrm{~mm}$ downstream with respect to the nominal target center. This forms a plane that defines the upstream edge of the space allowed for the FT. The first set of DCs is installed in front of the coils of the torus magnet, with an inclination of $65^{\circ}$ with respect to the beam axis. The front-end electronics boards of the DCs define the downstream border of the space allowance for the FT. The minimum distance of the DC boards from the beam axis is $\sim 140 \mathrm{~mm}$ at $2280 \mathrm{~mm}$ downstream with respect to the nominal center of the target. Taking into account the outside radius of the FT, including its insulation and the inclination angle of the DCs, the downstream face of the FT cannot exceed $\sim 2150 \mathrm{~mm}$ with respect to the nominal center of the target.

The FT needs cabling and service routing for the gas and cooling lines. These services must be connected to the outside of CLAS12. All services are installed in the shadow area of the torus magnet coils, i.e. in the six azimuthal slots extending radially from the beamline to the periphery. Each coil is $\sim 100$-mm thick, which allows space to

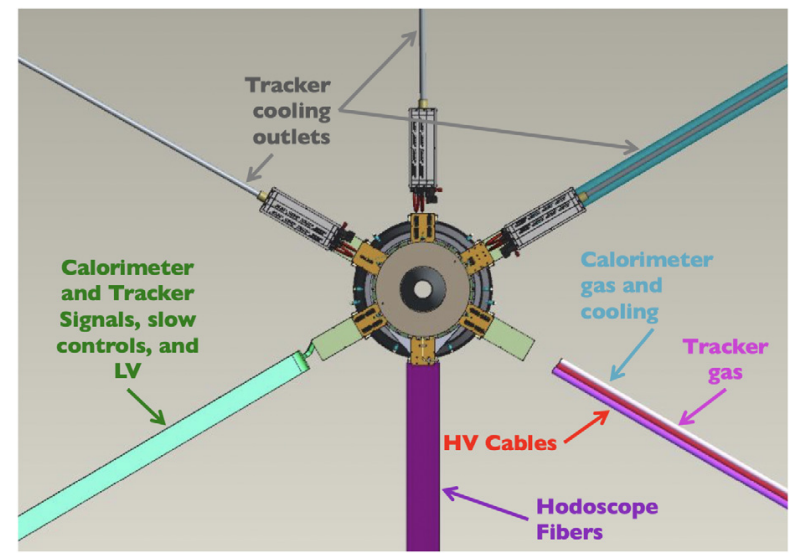

Fig. 14. Front view of the Forward Tagger with the routing of cables and services along the CLAS12 torus coils.

host some front-end electronics for the FT, which must be close to the detectors.

The whole FT is attached to the torus magnet cryostat by a support structure with flanges on both ends. This is needed both for the mounting sequence constraints and to avoid massive supports in front of the DCs. The support structure consists of two concentric stainless-steel pipes connected by adjustment screws to allow for precise alignment and positioning of the detector with respect to the beamline and the target position. A third tungsten cylinder of smaller diameter is located inside the steel pipes to provide shielding from beam background.

The FT is attached to the support structure via an inner tungsten pipe that is part of the calorimeter assembly and is located inside the central bore of the FT detectors. This pipe is designed to support the entire weight of the FT detectors and the additional shielding that is mounted upstream of the FT. Tungsten was chosen as the material because, even if less resilient, is more rigid than stainless steel, thus reducing the gravitational sagging, and has higher density and atomic number, i.e. better shielding properties. The FT-Cal is kept in position with respect to the inner tungsten pipe via four radial supports, made of PEEK. PEEK was chosen because of its low thermal conductivity $(0.25 \mathrm{~W} / \mathrm{mK})$ and its relatively high tensile strength ( $100 \mathrm{MPa})$. In addition, it features high radiation hardness and excellent stability over a broad range of temperatures. Mounting rings of PEEK and aluminum, respectively, are used to support and align the FT-Hodo and FT-Trk on the inner tungsten pipe.

Upstream of the FT, a tungsten cone is attached to the inner tungsten pipe to provide shielding from Møller electrons produced by the interaction of the beam in the target [16]. Fig. 2 shows a section of CLAS12 with the FT in its operating position.

\subsection{Routing of cabling and services}

All services and cables necessary for the operation of the FT detectors are routed along the torus coils to minimize the interference with the CLAS12 Forward Detector as shown in Fig. 14. These include cables for signals, $\mathrm{HV}, \mathrm{LV}$, and slow controls, as well as piping for gas distribution and cooling of the three FT subsystems.

The cables and piping are routed along the direction of the magnet coils using appropriate rails. The width and depth of the rails was chosen to be compatible with the space occupied by the DCs (both during normal operation and maintenance) and the clearance between the HTCC and the CLAS12 Forward Detector. 


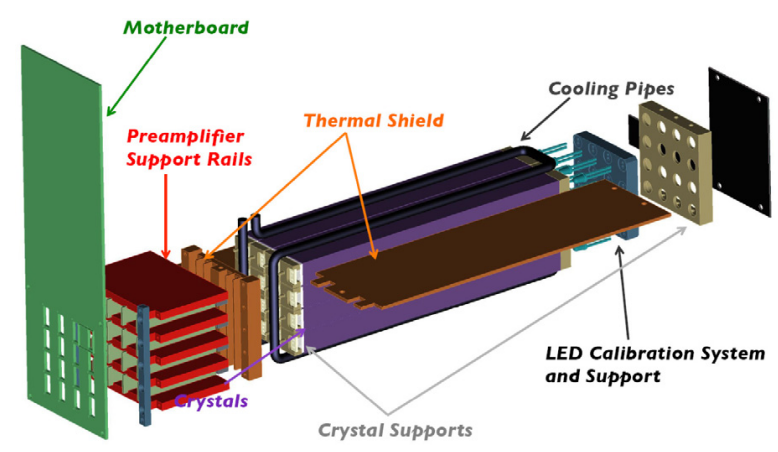

Fig. 15. Exploded view of the Proto-16 assembly. From left to right, the CAD drawing shows the motherboard, the system of copper rails holding the preamplifiers, the copper shield back plate, the crystal assembly, the copper shield front plate, and the LED board.

\section{FT prototypes}

Two prototypes of the FT-Cal, with 9 and 16 channels, respectively, were designed, assembled, and tested with cosmic rays and electron beams to optimize and validate the detector design. Specifically, the prototypes were used to check the single crystal mechanical assembly, the thermal performance, the front-end and read-out electronics, and the electrical connections via a motherboard. The response to cosmic rays was studied for both prototypes, while the response to electromagnetic showers was studied at Jefferson Lab (JLab) and the INFN Laboratory Nazionali di Frascati (LNF) in Italy. The 9-channel prototype (Proto -9 ) was tested at JLab using 2-3 GeV electrons deflected by the Hall B tagger system [16], while the 16-channel prototype (Proto-16) was tested at the Beam Test Facility of LNF with a $0.5 \mathrm{GeV}$ electron beam. Extensive simulations were performed and compared to the results of the two sets of measurements. The main goals of the tests were:

- to measure the energy resolution as a function of the single-crystal threshold;

- to measure the energy resolution as a function of $T\left(+18{ }^{\circ} \mathrm{C}, 0{ }^{\circ} \mathrm{C}\right.$, $\left.-10^{\circ} \mathrm{C},-25^{\circ} \mathrm{C}\right)$;

- to measure the time resolution;

- to verify the system linearity;

- to check rate performance;

- to validate Monte Carlo (GEMC) [17] simulations;

- to measure the electronic noise in realistic conditions;

- to perform detailed studies of the electromagnetic shower signal: shower profile, APD signal shape, and test the filtering algorithm.

The FT-Cal Proto-16 was built assembling $16 \mathrm{PbWO}_{4}$ Type-II crystals in a $4 \times 4$ matrix ( 8 provided by the BTCP and 8 from the RIINC company). Fig. 15 shows the Proto-16 components. Many mechanical and electrical solutions tested on Proto-16 were then adopted in the final FT-Cal design. Due to the significant size of the crystal matrix, the expected performance of Proto-16 in terms of energy resolution for showers generated at the center of the $4 \times 4$ matrix is similar to what was expected for the FT-Cal. Proto-16 was tested at the Beam Test Facility (BTF) [18] of LNF, using a $0.5 \mathrm{GeV}$ electron beam. Data were taken in October 2012 to study the prototype resolution as a function of the energy deposition and the calorimeter temperature. The BTF electron beam is characterized by a repetition frequency of $50 \mathrm{~Hz}$ and a pulse duration of $10 \mathrm{~ns}$. The beam intensity can be varied by operating different sets of slits, selecting the number of electrons per bunch at the level of a single particle. The prototype performance could therefore be studied as a function of the number of electrons simultaneously hitting the crystal matrix, i.e. of the detected energy.

Fig. 16 shows the BTF experimental hall after the installation of Proto-16 and the associated equipment. The detector was placed on

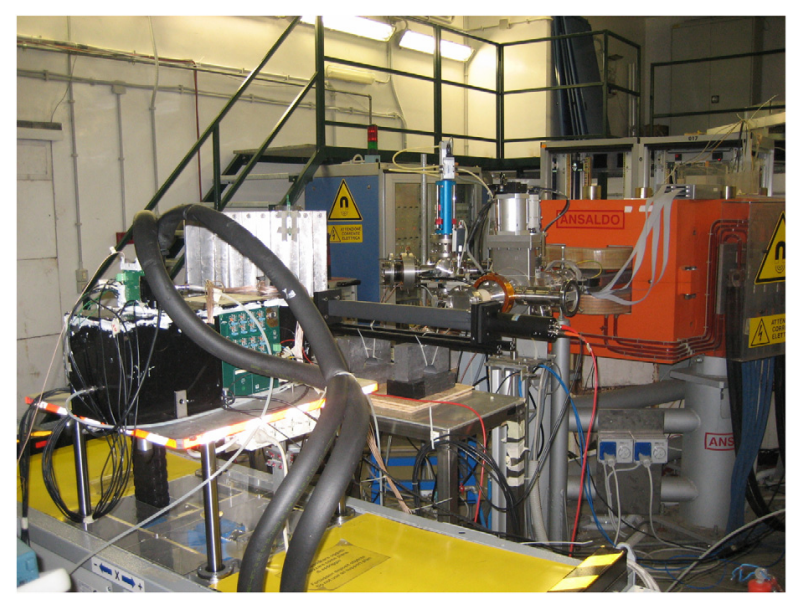

Fig. 16. Experimental setup of the Proto-16 test at the LNF Beam Test Facility (BTF). The beam comes from the right. On the left, the detector inside its case (black) is placed on a movable table to allow for centering of the calorimeter with respect to the beam. In front of the calorimeter, a plastic scintillator bar wrapped in black Tedlar is used to determine the arrival time of the beam electrons.

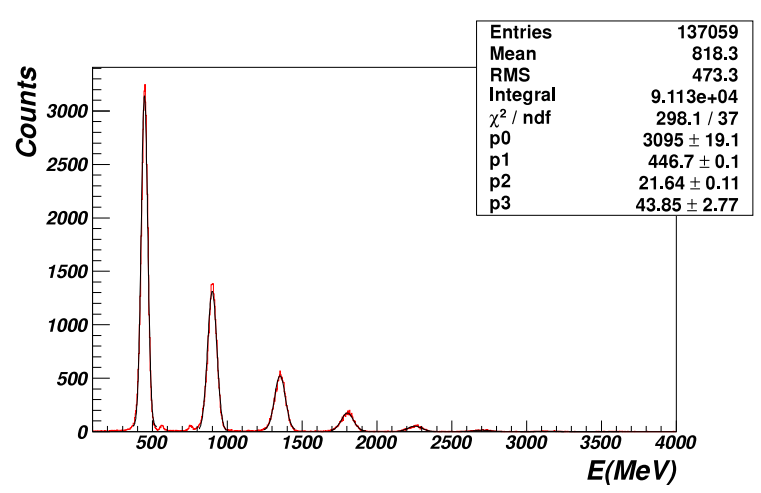

Fig. 17. The total energy measured by Proto- 16 after calibration. The peaks correspond to different bunch populations and are clearly visible and well separated.

a movable table that could be displaced in the $x$ and $y$ directions (transverse plane) with a $0.1-\mathrm{mm}$ accuracy. This feature was exploited to center the calorimeter with respect to the beam. A plastic scintillator bar, read out by two PMTs, was placed in front of the beam pipe exit window and was used to determine the arrival time of the electron within the 10-ns bunch duration. The data acquisition system, based on the JLab CODA standard [3], was triggered by the radio-frequency (RF) signal of the Frascati accelerator. For each trigger all of the signals of the Proto-16 crystal matrix and of the scintillator-bar PMTs were recorded by CAEN VME boards. Both the Proto-16 and scintillator signals were sent to a passive splitter whose two outputs were connected to the $250 \mathrm{MHz}$ FADCs and to leading-edge discriminators. The discriminator output was sent to pipeline TDCs. The samples recorded by the FADCs in an 800 ns window were recorded for each trigger and analyzed offline to evaluate the charge and time.

The conversion between charge and energy was first determined using cosmic ray measurements and then optimized by studying the response of each crystal to $0.5 \mathrm{GeV}$ electrons at the LNF-BTF. It is worth noting that the new calibration constants were found to be within 5\%$10 \%$ of the initial values determined during cosmic-ray data taking. The total reconstructed energy after the full calibration is shown in Fig. 17 for an electron multiplicity on the order of 1-2. The peaks corresponding to different bunch populations are clearly visible and well separated.

Energy resolution. The mean values and widths $(\sigma)$ of the peaks in the total reconstructed energy spectrum were analyzed to check the 


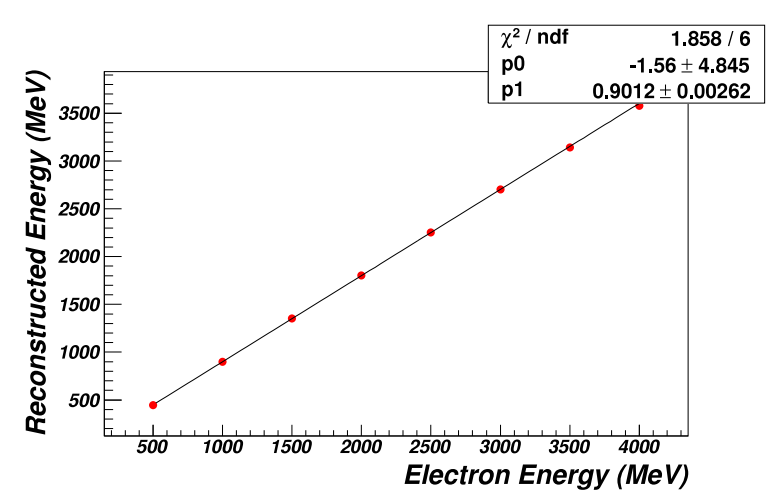

Fig. 18. Proto-16 reconstructed energy as a function of the beam bunch energy. The red points were obtained at room temperature and with an APD gain of 150. The linear regression of the experimental points shows no deviation from linearity.

system linearity and to determine the resolution. The measurements were performed by centering the beam on the calorimeter to have the maximum containment of the electromagnetic shower. Fig. 18 shows the fitted peak position as a function of total energy in the beam bunch for an APD gain of 150 and a $\mathrm{PbWO}_{4}$ temperature of $18{ }^{\circ} \mathrm{C}$. The linear regression of the experimental points shows no deviations from linearity in the explored range. The same measurement performed in different experimental configurations gave consistent results, confirming that the system is linear up to the maximum measured energy of $4 \mathrm{GeV}$.

Fig. 19 shows the energy resolution as a function of the energy in the beam bunch. The colored points correspond to the resolution measured with Proto-16, while the black open circles are the results of the Monte Carlo (GEMC) simulations. The error bars in the graph show the statistical uncertainty, while the systematic uncertainty was estimated to be on the order of $5 \%$. As expected, the experimental resolution improves for increasing energy, reaching an asymptotic behavior at about $3 \mathrm{GeV}$. The measurements performed in different configurations are in general consistent, varying within a range of $0.5 \%$ except for the resolution obtained at room temperature and $G=75$ (orange points). The resolution in this case is systematically worse than that obtained at the same temperature but $G=150$. This was interpreted as due to the preamplifier noise being the dominant factor in determining the resolution at this temperature. From this we concluded that working at higher APD gain is the preferable configuration.

The comparison of the resolutions obtained at different temperatures shows that lower temperatures, corresponding to higher light yield, and therefore a larger signal, give a better resolution. The best values were obtained at $-20^{\circ} \mathrm{C}$, where the experimental points are in good agreement with the simulation results. The dependence of the resolution on the temperature is more evident for high bunch energies, where threshold effects are smaller. Above $2 \mathrm{GeV}$, the resolution at room temperature seems to be systematically higher than that obtained at $0{ }^{\circ} \mathrm{C}$ or $-20{ }^{\circ} \mathrm{C}$ with a difference of about $0.5 \%$. The difference of the resolution obtained at $0{ }^{\circ} \mathrm{C}$ and $-20{ }^{\circ} \mathrm{C}$ is on the contrary negligible within the systematic uncertainties. Based on these results and considering the technical difficulties in operating the FT-Cal at the lowest temperature, we chose the optimal operating temperature of the calorimeter to be $0{ }^{\circ} \mathrm{C}$.

\section{Detector simulations}

Detailed simulations of the FT have been done with the Geant4based Monte Carlo code for CLAS12, GEMC [17], to optimize the detector design, to develop the reconstruction algorithms, and to understand the detector performance.

Details on the implementation of the FT in GEMC of the detector geometry and digitization are reported in Ref. [17], while an extensive

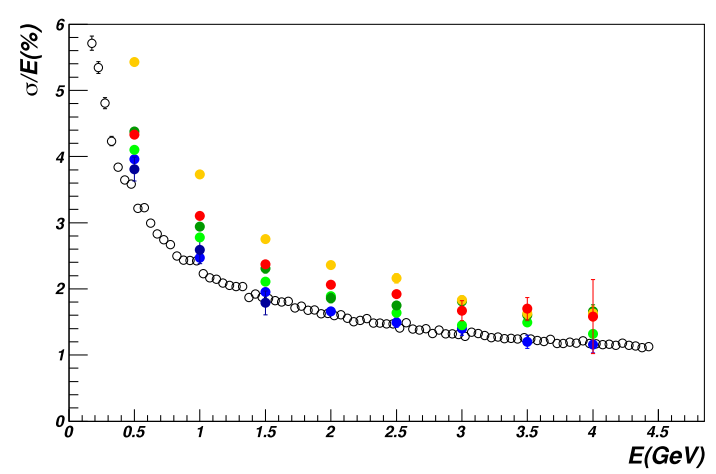

Fig. 19. Proto-16 energy resolution as a function of the beam bunch energy. The red and orange points were obtained at room temperature for APD gains of 150 and 75, respectively. The green points correspond to $0{ }^{\circ} \mathrm{C}$; the darker points were obtained removing the passive splitter. The blue and dark-blue points, that partially overlap, correspond to $-20{ }^{\circ} \mathrm{C}$ with APD gains of 150 and 75 , respectively. The open black circles show the expected resolution based on Monte Carlo simulations. Only statistical uncertainties are shown. (For interpretation of the references to color in this figure legend, the reader is referred to the web version of this article.)

discussion of the simulation studies that guided the detector design are presented in Ref. [14]. Here we focus on summarizing the results of the simulation studies that are relevant to understand the FT performance.

\subsection{Leakage corrections}

The reconstructed cluster energy can be systematically smaller than the actual energy of the particle that induced the shower due to leakages in the shower containment caused by the limited dimensions of the calorimeter, by cuts in the clustering algorithms, and by the thresholds in the hit detection. An example of the difference between the reconstructed cluster energy and the simulated electron energy is shown in the top panel of Fig. 20. This was obtained assuming an equivalent threshold on the individual crystals of $10 \mathrm{MeV}$ : the leakage varies from $\sim 80 \mathrm{MeV}$ (16\%) for $500 \mathrm{MeV}$ electrons to $\sim 300 \mathrm{MeV}(6.6 \%)$ for $4.5 \mathrm{GeV}$ electrons.

This effect can be easily corrected for by parameterizing the leakage as a function of the reconstructed cluster energy and position, and applying the correction in reconstruction. Simulations of single electrons were performed in GEMC and the difference between the reconstructed cluster energy and the electron energy was studied as a function of the cluster seed crystal (i.e. the crystal with the largest signal). For each crystal, the dependence of this difference on the reconstructed cluster energy was fit to a fourth-order polynomial, which was then used as an additive correction to the reconstructed cluster energy. The final dependence of the difference between the corrected cluster energy and simulated energy is shown in the bottom panel of Fig. 20.

\subsection{Electromagnetic background and radiation dose}

The electromagnetic background produced by the interaction of the electron beam in the target at the nominal CLAS12 luminosity was simulated in GEMC. For this purpose, in each event, about 124k, $11-\mathrm{GeV}$ electrons were generated that originated $10 \mathrm{~cm}$ upstream of the target. The electrons were distributed randomly with the radiofrequency structure of the beam in a 250-ns window. This number of electrons corresponds to the number of beam electrons that would pass through the target in the chosen time window at the nominal CLAS12 luminosity of $10^{35} \mathrm{~cm}^{-2} \mathrm{~s}^{-1}$. These simulations were used to study background rates in each of the FT detectors, to determine the pile-up probability, and to estimate the radiation dose the FT would be subject to during operations.

The overall particle rate in the FT was found to be about $120 \mathrm{MHz}$, dominated by very low-energy particles, with only $6 \%$ due to particles 

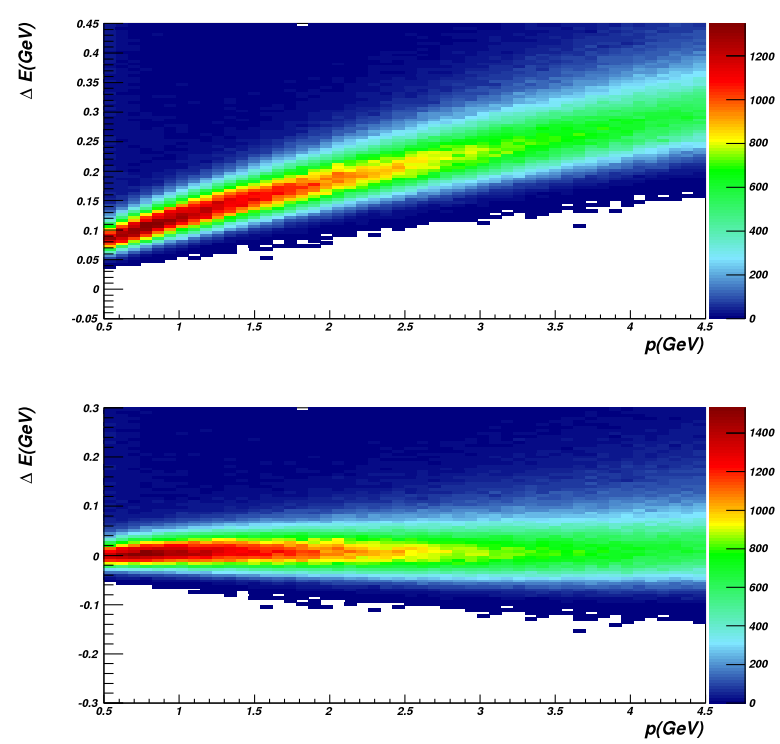

Fig. 20. Top: difference between the simulated electron energy and the reconstructed cluster energy as a function of the electron momentum for a $10 \mathrm{MeV}$ equivalent threshold on the single crystal signal. Bottom: difference between the simulated electron energy and the cluster energy after the leakage correction.

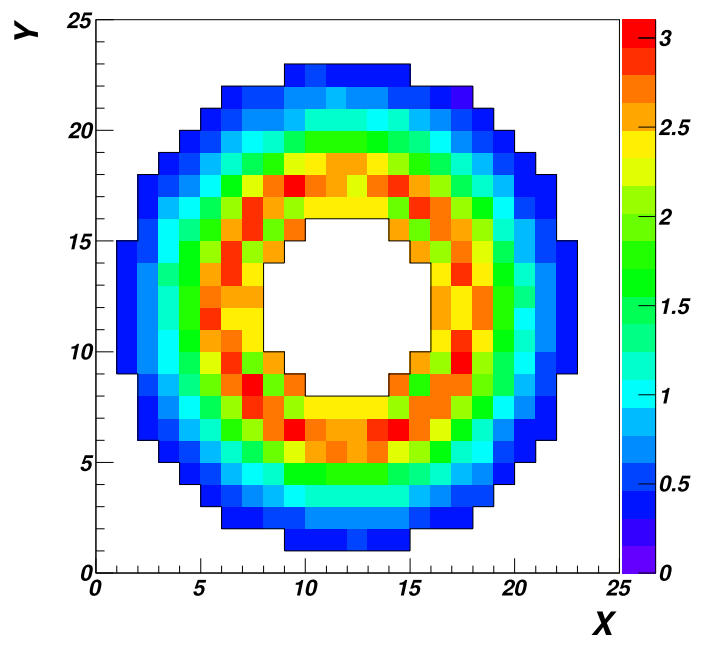

Fig. 21. Radiation dose on the FT calorimeter crystals in $\mathrm{rad} / \mathrm{h}$ at $10^{35} \mathrm{~cm}^{-2} \mathrm{~s}^{-1}$ luminosity. The maximum values of about $5 \mathrm{rad} / \mathrm{h}$ are observed for the innermost crystals, i.e. at the smaller angles.

with energy above $100 \mathrm{MeV}$. In the energy range to be tagged (0.5$4.5 \mathrm{GeV}$ ) the overall particle rate is further reduced to about $180 \mathrm{kHz}$, equally shared between photons and hadrons.

For the FT-Cal, the energy deposition in each crystal was evaluated from the background simulation and used to calculate the dose per unit of time. The overall radiation dose at $10^{35} \mathrm{~cm}^{-2} \mathrm{~s}^{-1}$ was estimated to be less than $1.5 \mathrm{rad} / \mathrm{h}$ when averaged over the entire calorimeter with a distribution on the calorimeter crystals as shown in Fig. 21. The maximum dose per crystal is about $3 \mathrm{rad} / \mathrm{h}$, which would result in a maximum integrated dose per crystal of about $2160 \mathrm{rad}$ in 30 days of beam time.

\section{Detector calibration and commissioning}

\subsection{Pre-beam calibration}

Initial checkout and calibration of the FT detectors upon completion of the installation were performed via:

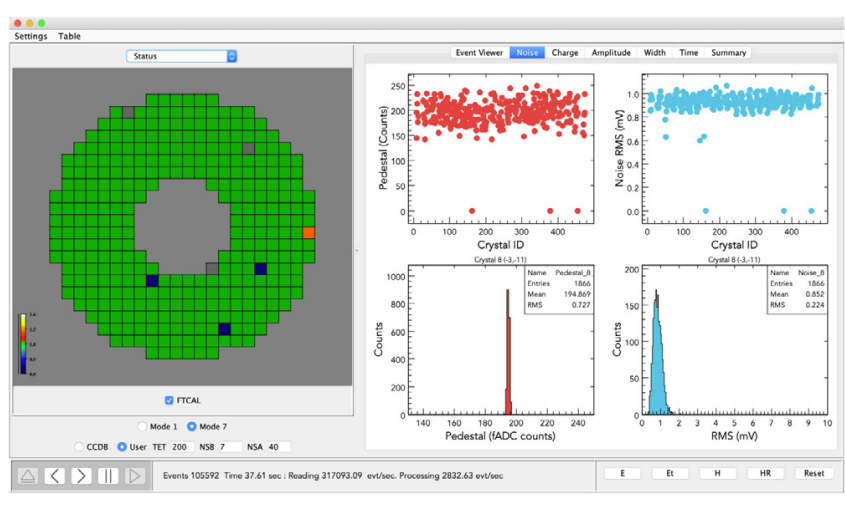

Fig. 22. Results of the FT-Cal noise analysis from a pulser run. The left part of the calibration suite display shows a view of the calorimeter with a color scheme representing the status of the crystal: green corresponds to a fully functional element, blue to an element with noise below the typical range (indicative of a low-gain preamplifier), orange to an element with noise above the typical range, and gray to a crystal for which no data were recorded. The right part of the panel shows the average pedestal and noise as a function of the crystal number, and the event distribution of the pedestal and noise for the selected crystal. (For interpretation of the references to color in this figure legend, the reader is referred to the web version of this article.)

- Pulser, LED, and cosmic ray runs for the FT-Cal;

- Pulser and cosmic ray runs for the FT-Hodo;

- Pulser and pedestal runs for the FT-Trk.

\subsubsection{FT-cal pre-beam calibration}

Initial checkout of the calorimeter was performed via pulser and LED runs. In the pulser runs, an external clock was used to trigger the readout of the entire FT-Cal recording the full FADC waveforms in a 400-ns window in the absence of a physics signal to measure baselines and to monitor noise, for the purpose of identifying disconnected or malfunctioning channels. For each crystal, several parameters were studied, such as the average pedestal, the event-by-event pedestal RMS, and the noise defined as the sample-by-sample pedestal RMS. The analysis was performed online, connecting to the data acquisition Event Transfer (ET) ring [3], or from a recorded data file using the FT Java calibration suite [19]. Fig. 22 shows a view of a typical pulser run analysis. One the most useful results obtained from this analysis is the average channel noise that is indicative of its functionality: a noise level below the typical range is indicative of a malfunctioning preamplifier or a disconnected cable, while a noise level above the typical range can indicate a high-voltage issue since the noise introduced by the LAAPDs is higher when the biased voltage is not applied.

Once the initial debugging of the system based on pulser runs was completed, a second checkout based on LED runs was performed. In this case, the FT-Cal LMS was used to input light into each of the calorimeter crystals and the corresponding signals were recorded to check the pulse amplitude and shape, and to assess the correct functioning of the LAAPDs, preamplifiers, and front-end electronics. Using the EPICS slow controls interface of the LMS, the LEDs can be switched on in groups of 6 , one per driver, in a predefined sequence and pulsed at a rate of $62.5 \mathrm{~Hz}$ for a time interval of $30 \mathrm{~s}$ to accumulate about 1800 waveforms per channel. The LED pulse amplitudes have been tuned to provide a maximum amplitude at the FADC of about $1 \mathrm{~V}$, which is representative of a typical signal expected for the calorimeter. The recorded waveforms are analyzed to extract the pulse amplitude as a function of time. In fact, upon being turned on, the LED light intensity undergoes an exponential drop until it reaches stability. This typically happens within 6-8 $\mathrm{s}$. The amplitude in the stability region is fit to a constant to extract the average value that is recorded and compared to reference values to detect changes in the detector response and potential failures. Fig. 23 shows the results of the analysis of a typical LED run as displayed by the calibration suite. In this specific 


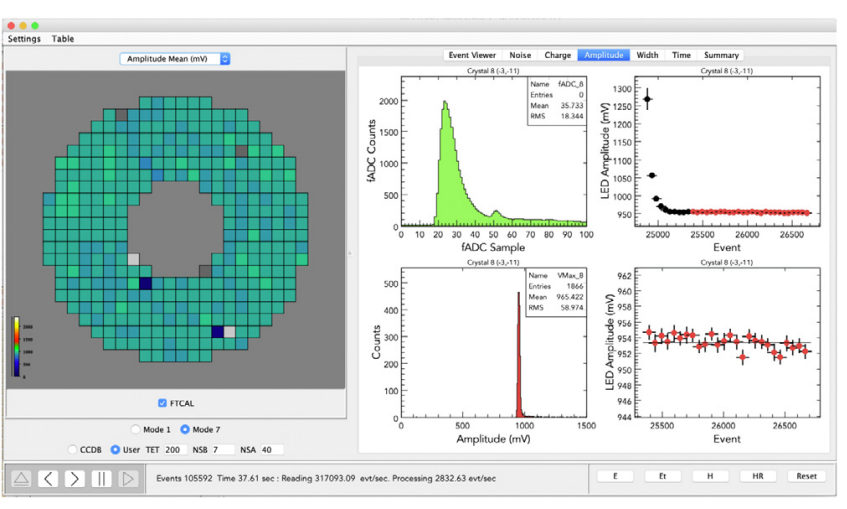

Fig. 23. Results of a typical FT-Cal LED run. The left part of the calibration suite display shows a view of the calorimeter with a color scheme representing the LED pulse amplitude. The right part of the panel shows for the selected crystal the average pulse shape (top left), the pulse amplitude as a function of the event number, i.e. of time (top right), the distribution of the amplitudes (bottom left), and the pulse amplitude as a function of the event number after the LED has reached stability (bottom right). The latter is fit to a constant to determine the pulse amplitude that is displayed in the detector view.

case, the analysis shows a relatively uniform response to the LED light, with typical amplitudes on the order of $1 \mathrm{~V}$ as defined by the design, with a few problematic channels that coincide with those identified by the pulser runs of Fig. 22.

The final calibration of the FT before in-beam testing was based on the study of the detector response to cosmic rays. A special FPGAbased trigger was developed by the JLab Fast Electronics Group to select events where a cosmic ray crosses the calorimeter primarily in the vertical direction, i.e. crossing the crystals along the short side. This is achieved by requiring a minimum number of signals above threshold in the crystals that are in a "column" of the calorimeter assembly, a technique that exploits the functionalities of the JLab FADCs and trigger electronics $[3,20]$. For these events, the waveforms for all crystals in the calorimeter were recorded and analyzed offline using the FT-Cal calibration suite. Details of the analysis procedure are reported in Refs. [21,22]; here we summarize only the main steps and results. For each crystal, events where at least $N_{\min }$ crystals with signal above threshold are found in a vertical range of $N_{\text {range }}$ crystals above or below the chosen one were selected. After optimization, the values of $N_{\min }$ and $N_{\text {range }}$ were fixed to 4 and 5, respectively. For these events, the crystal waveform was integrated in a fixed range and pedestal subtracted to extract the charge. The integration range was optimized empirically to maximize the signal-to-noise ratio. The charge distribution for all selected events in the given crystal was then fit with a Landau summed with an exponential function, representing the minimum-ionizing particle (MIP) deposition and background, respectively. The mean of the Landau function, compared with the expected average energy deposition determined from Geant4 Monte Carlo simulations to be $15.3 \mathrm{MeV}$, was then used to evaluate the charge-to-energy conversion factor for each crystal.

Fig. 24 shows an example of a cosmic ray event as displayed by the calibration suite and an example of the charge distribution for a selected crystal obtained by integrating over the selected events. The typical values of the Landau peak were found to be in the range of 4-7 pC at the calorimeter operating temperature of $0{ }^{\circ} \mathrm{C}$ and the corresponding conversion factors in the range of $2.2-3.8 \mathrm{MeV} / \mathrm{pC}$. These values were used as the calibration constants for the initial reconstruction of beam data, although it was found that these constants usually led to an overestimate of $20 \%$ of the actual energy deposited in the energy range of interest for the calorimeter of $0.5-4.5 \mathrm{GeV}$. While this discrepancy is significant, it is not unexpected given the uncertainties in extracting the cosmic ray signal from the background and the large difference in the two calibration points, since cosmic rays deposit an energy in the range of tens of $\mathrm{MeV}$, while the energy range for beam-induced signals is two orders of magnitude larger.
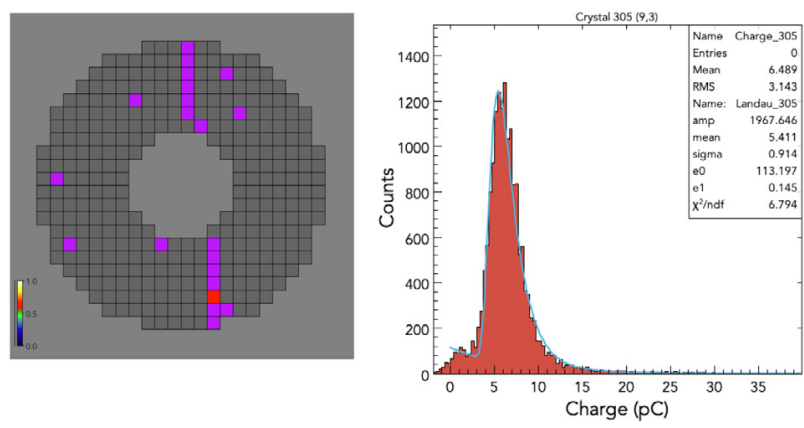

Fig. 24. Left: example of a cosmic ray crossing the calorimeter vertically as displayed by the calibration suite. Right: example of the measured charge distribution measured from the selected events for a calorimeter crystal; the blue line shows the results of the Landau plus exponential fit; the mean of the Landau function is used to estimate the charge-to-energy conversion factors. (For interpretation of the references to color in this figure legend, the reader is referred to the web version of this article.)

\subsubsection{FT-Hodo pre-beam calibration}

Similarly to the calorimeter, initial checkout of the hodoscope was performed via pulser runs to check the functionality of each electronics channel and to evaluate the SiPM gains by measuring the single photoelectron (SPE) signal. An external clock was used to trigger the data acquisition, which recorded the waveform of all 232 channels in a $400 \mathrm{~ns}$ window. The waveforms could be analyzed online by connecting the calibration suite to the data acquisition ET ring [3] or offline reading from the data file. The parameters that were monitored are the pedestal values, the pedestal RMS, and the electronic noise. The extracted SPE values were compared to the typical ones to identify problematic channels and disconnected cables. For each channel, the waveforms that exceeded a minimum threshold above the baseline were analyzed to extract the SPE signal. For this purpose, the waveforms were integrated in a fixed time range and pedestal subtracted. The distribution of the extracted charge for a selected channel is shown in Fig. 25, where the top and bottom plots are for the same tile in the two detector layers and the left and right plots show the results obtained using the waveform maximum and integral, respectively. Clear peaks corresponding to one, two, and three photoelectrons are visible; the difference between the peaks was used to determine the gain of the channel, resulting in typical values on the order of $20 \mathrm{pC} / \mathrm{phe}$. The consistency of the results obtained using the pulse maximum and integral confirms the reliability of the waveform analysis.

Further checkout of the detector was performed via cosmic ray data taking. The same FPGA-based trigger developed for the calorimeter was used to trigger the data acquisition system on events in which multiple tiles of the hodoscope had a signal above threshold. For such events, all hodoscope channel waveforms were recorded and analyzed offline. The signal charge was extracted by integrating the waveform in a fixed time window and subtracting the pedestals. The resulting charge distributions were inspected to ensure a sizable signal for all tiles. In this case no attempt was made to extract the charge-to-energy conversion factor from these distributions because of the unfavorable orientation of the hodoscope in the installation position for the measurement of cosmic rays that could cross the scintillation tiles with a very large angular and energy deposition spread.

\subsubsection{FT-Trk pre-beam calibration}

The first calibrations and tests of the trackers were performed using the cosmic-ray test bench available at CEA-Saclay [15]. The goal of these tests was to optimize the operating conditions of the detectors and to compute their two-dimensional efficiency maps using cosmic muons prior to shipment to JLab. Fig. 26 shows the results for two of the four detector layers, indicating a good uniformity of the response over the full active area. 

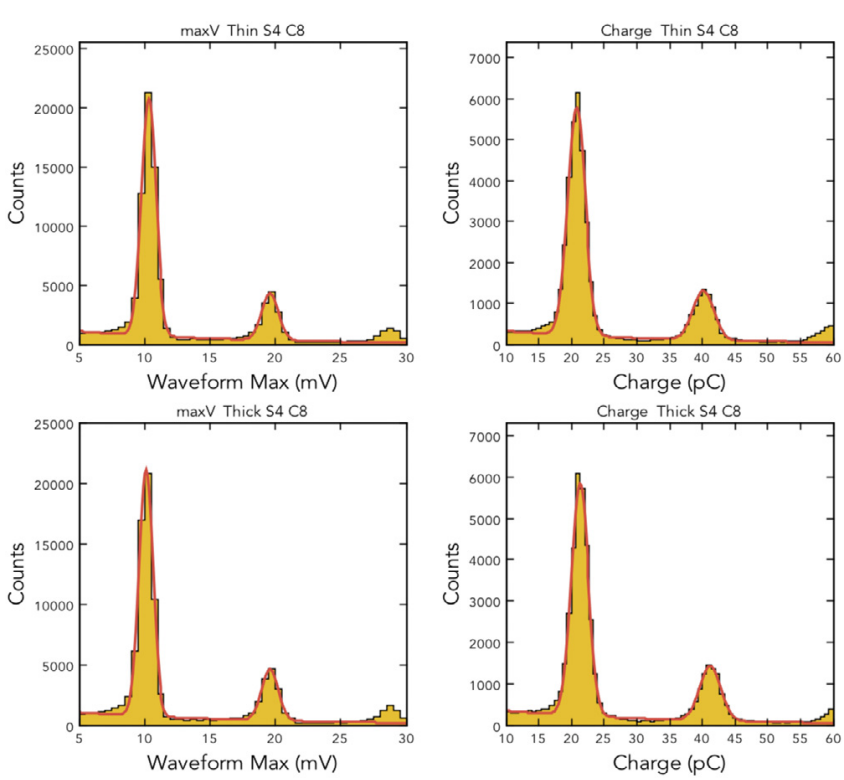

Fig. 25. SPE signal from the FT-Hodo SiPMs reading signals from the thin (top) and thick (bottom) tiles, in $\mathrm{mV}$ (left) and $\mathrm{pC}$ (right), determined using the waveform maximum and integral, respectively.
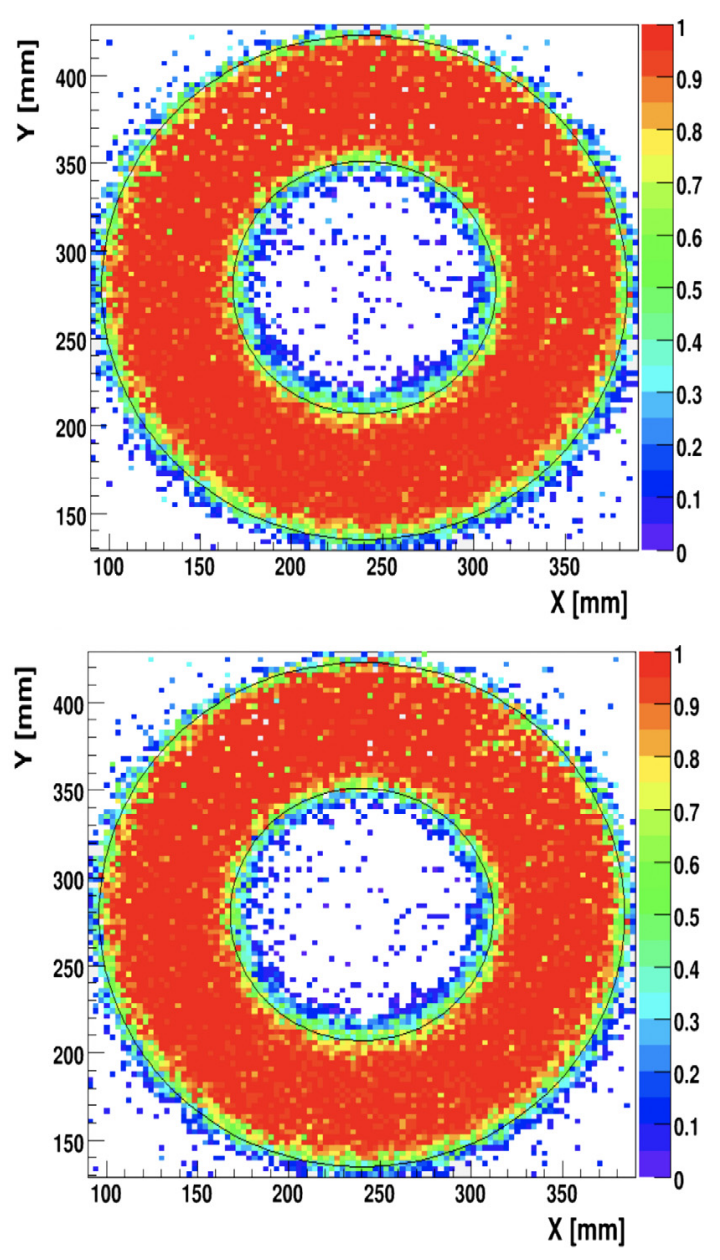

Fig. 26. Two-dimensional ( $y$ vs. $x$ coordinate) efficiency map for the two layers of one of the FT tracker detectors as measured in the cosmic-ray setup at CEA-Saclay. The black circles indicate the limits of the detector active area.

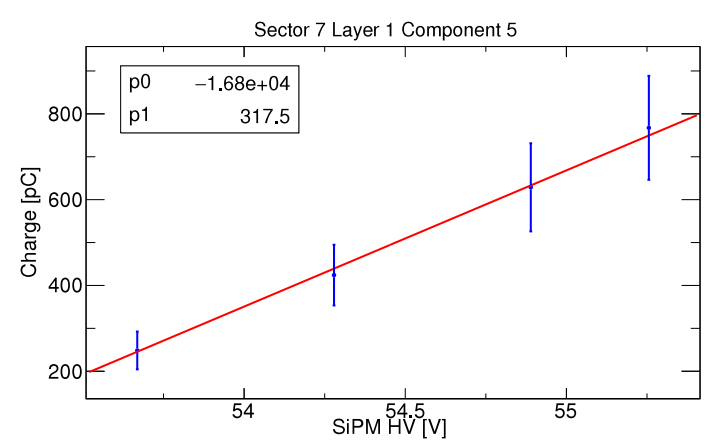

Fig. 27. Dependence of the MIP mean position on the SiPM bias voltage for a single hodoscope tile. The dependence is fit to a linear function that is used to select the operating voltage to give an average MIP signal close to the chosen value.

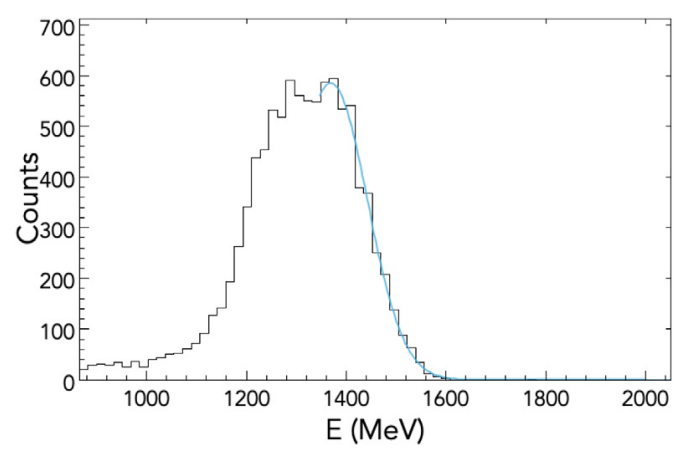

Fig. 28. Example of the seed energy distribution for a selected crystal for elastic events at $2.2 \mathrm{GeV}$ beam energy. The blue line shows the fit used to determine the edge of the distribution. (For interpretation of the references to color in this figure legend, the reader is referred to the web version of this article.)

After installation, the initial checkout of the FT-Trk and, in particular, of the front-end electronics, was performed by means of pedestal and pulser runs. Since these procedures are standard for the CLAS12 Micromegas detectors, we refer to Ref. [15] for further details.

\subsection{In-beam calibration and commissioning}

While pre-beam calibrations were essential to ensure all detector components were fully operational, the final calibrations to extract the parameters needed for the FT reconstruction are based on analysis of beam data. Here we report specifically on the procedures developed for the calibration of the calorimeter and hodoscope, since no specific calibrations are needed for the tracker.

For both the hodoscope and calorimeter, energy and time calibrations can be obtained from the analysis of data recorded with the CLAS12 production triggers and do not require dedicated data taking. A dedicated run is typically employed, however, for matching the gains from all FT-Hodo SiPMs. ${ }^{6}$ In this dedicated run, average minimumionizing particle signals were obtained for a set of different HV settings (see Fig. 27), determining the slope and intercept from which gain matching is established.

The energy calibration for the FT-Cal is achieved by analyzing electron elastic scattering events or by reconstructing the $\pi^{0} \rightarrow \gamma \gamma$ decay where both photons are detected in the calorimeter.

Elastic $e p \rightarrow e p$ scattering data were found to be particularly effective for calibrations at low beam energy. Data using a $2.2 \mathrm{GeV}$ beam were collected during the CLAS12 engineering run. Events with only one cluster in the FT-Cal were selected (from the scattered electron)

6 Having a matched gain from all FT-Hodo SiPMs allows for a common trigger readout threshold for all channels. 

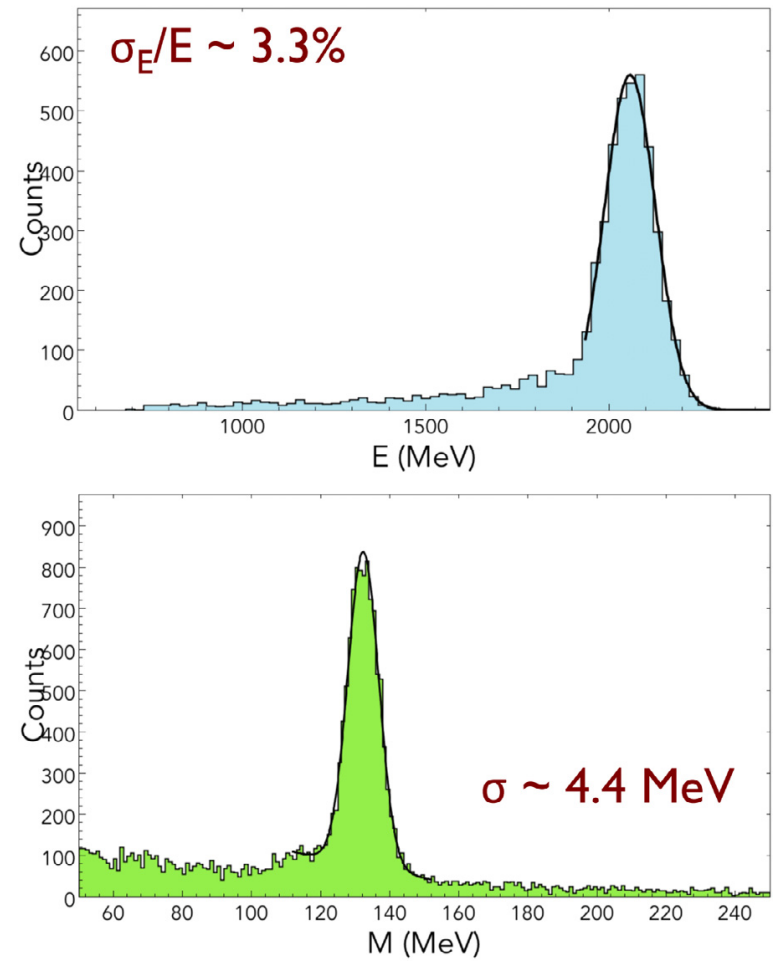

Fig. 29. Top: electron energy spectrum reconstructed at $2.2 \mathrm{GeV}$ beam energy in the FT-Cal; the peak corresponds to elastic scattering; after calibrations based on elastic events, an overall energy resolution of $3.3 \%$ at $2.2 \mathrm{GeV}$ is found. Bottom: $\pi^{0} \rightarrow \gamma \gamma$ invariant mass spectrum reconstructed at $10.6 \mathrm{GeV}$ beam energy using the elastic scattering energy calibrations: the width of the $\pi^{0}$ peak determined via a Gaussian fit was found to be $\sim 4.4 \mathrm{MeV}$.

and, based on the existing cosmic ray calibrations, the energy of the crystal with the largest signal, i.e. the seed, was extracted. For each crystal, these events were accumulated requesting the seed energy to be larger than $55 \%$ of the total cluster energy. The right edge of the distribution of the seed energy was fit with a Gaussian function to extract the peak position. The mean value of the Gaussian function was compared to that expected based on Geant4 Monte Carlo simulations to extract a correction to the charge-to-energy conversion factor used in the cluster reconstruction. Fig. 28 shows an example of the seed energy distribution and the cluster energy distribution for a selected crystal. Using these constants, an energy resolution of $3.3 \%$ at $2.2 \mathrm{GeV}$ beam energy was determined by fitting the reconstructed elastic peak (see Fig. 29). This resolution is about $1 \%$ larger than what is expected from simulations as discussed in Section 8. With the same calibration constants, the $\pi^{0} \rightarrow \gamma \gamma$ decay was reconstructed at $10.6 \mathrm{GeV}$ beam energy selecting events with both photons detected in the FT-Cal, finding the width of the $\pi^{0}$ peak to be $\sim 4.4 \mathrm{MeV}$, which gives an energy resolution of $\sim 3.2 \%$.

Since the effectiveness of the elastic calibration is limited to beam energies on the order of a few $\mathrm{GeV}$ because of the rapid decrease of the corresponding cross section at higher energies, an alternative approach was developed to perform the energy calibration of the FT-Cal based on $\pi^{0} \rightarrow \gamma \gamma$ decays. Events where both photons are detected in the calorimeter were selected and filtered applying the following cuts:

- the energy of both clusters, as reconstructed based on existing calibrations, is larger than $500 \mathrm{MeV}$;

- the size of both clusters, i.e. the number of crystals involved, is larger than 3;

- the opening angle between the two clusters is larger than $2^{\circ}$.

The last cuts are useful to reduce backgrounds resulting from split clusters, i.e. events in which a secondary particle originating from the
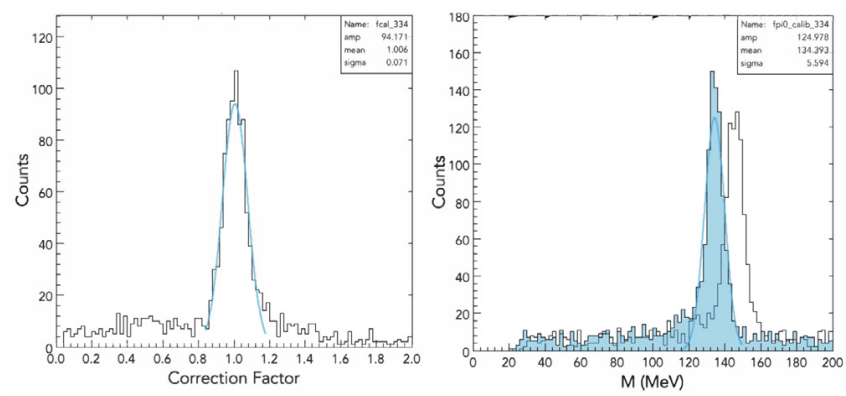

Fig. 30. Left: calibration correction factor for a selected crystal computed as the ratio between (1) the measured energy of clusters where the crystal is the seed and the energy calculated from the nominal $\pi^{0}$ mass and (2) the other cluster energy. Right: $\pi^{0}$ mass spectrum for the same crystal before (unfilled histogram) and after (filled histogram) the calibration procedure.
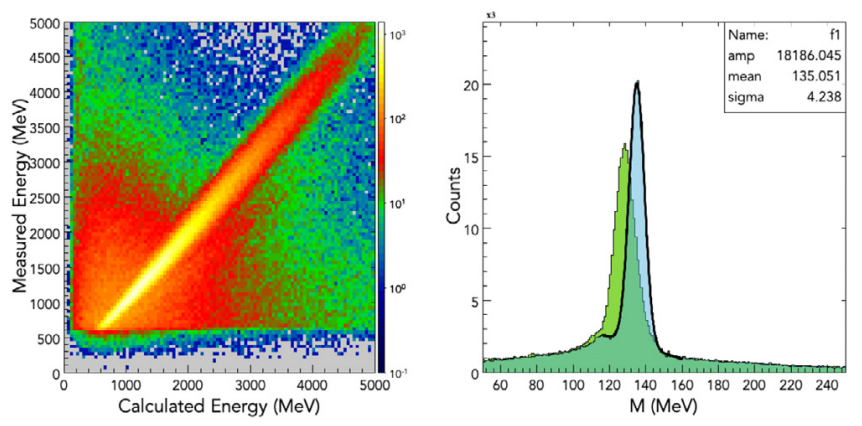

Fig. 31. Left: correlation between the measured cluster energy and the energy computed from the nominal $\pi^{0}$ mass; the range covered is well matched to the FT energy range of interest. Right: $\pi^{0}$ mass spectrum before (green) and after (blue) the calibration; the achieved resolution is $\sim 4.2 \mathrm{MeV}$. (For interpretation of the references to color in this figure legend, the reader is referred to the web version of this article.)

electromagnetic shower creates a second cluster at a close distance to the primary cluster. For each crystal, events in which the crystal is the seed of one of the two clusters are accumulated and the ratio between (1) the measured cluster energy for the given crystal and the energy calculated from the nominal $\pi^{0}$ mass and (2) the other cluster energy is computed. The distribution of such ratios is fit with a Gaussian function to derive a correction factor for the charge-to-energy calibration constant of the selected crystal. The procedure is applied iteratively until the $\pi^{0}$ mass spectrum for all crystal is within $0.5 \mathrm{MeV}$ of the nominal value.

Fig. 30 shows an example of the ratio distribution and of the $\pi^{0}$ mass spectrum for a selected crystal before and after (blue histogram) the calibration procedure. The advantage of this procedure is that it does not strongly depend on the beam energy and exploits the full energy spectrum of the clusters, providing a check of the linearity. The left panel of Fig. 31 shows the correlation between the measured and computed cluster energies after calibration: the energy range, which is covered with good statistics, is from 0.5 to $5 \mathrm{GeV}$ with a perfect overlap with the energy range of interest for the CLAS12 experimental program with the FT. The resolution that is achieved with this calibration algorithm is of the order of 4-5 MeV integrated over the entire calorimeter as shown by the right panel of Fig. 31 .

The energy calibration of the FT-Hodo is performed by studying the energy deposition of MIPs, since these are the typical signals expected from charged particles impinging on the detector. Fig. 32 shows the charge from MIP signals in the thin and thick tiles. For the FTHodo, charged particle signals are selected by requiring the geometrical matching of tiles in the two layers. No other requirement or matching with other detectors is requested to minimize the dependency on other system calibrations. The distributions are fit with a Landau plus an 

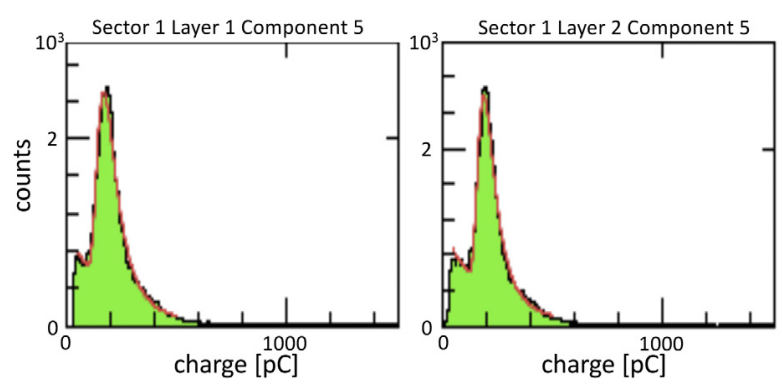

Fig. 32. Signals from two FT-Hodo tiles (thin and thick layer) fit with a Landau plus an exponential to established the charge-to-energy constants.
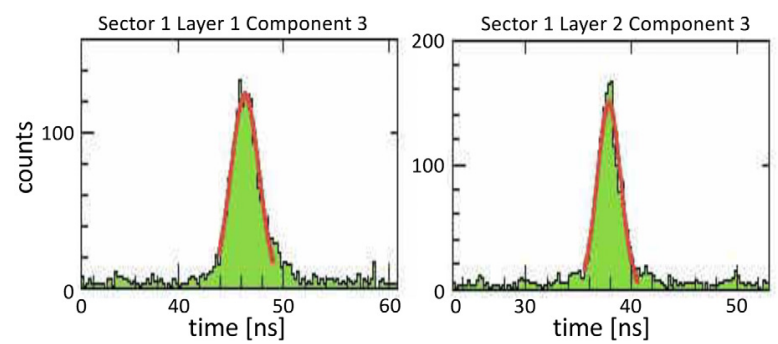

Fig. 33. FT-Hodo time corrections determined by Gaussian fits on the time difference between the hit time projected back to the event vertex and the event start time for a thin (left) and thick (right) tile.

exponential function to determine the average MIP charge. The chargeto-energy conversion factors are determined by comparing the resulting values to the ones estimated from Geant4 Monte Carlo simulations. The constant values were found to be very stable with time, requiring the calibration to be performed only at the beginning of a new data taking period or after a change of the detector operating conditions (e.g. a change of the HV settings).

The timing calibrations of both the FT-Cal and FT-Hodo are obtained by studying the time correlation of the signals in the two detectors with the CLAS12 Forward Time-of-Flight (FTOF) detector [23]. The procedure makes use of events with a scattered electron in the CLAS12 Forward Detector and a second particle detected in the FT. In such events, the start time $t_{0}$, i.e. the time of the interaction of the beam electron in the target, can be computed from the electron FTOF time projected back to the event vertex. The start time can then be used as a reference for the calibration of the FT detectors.

For the FT-Hodo, the signal time, $t_{h i t}$, projected back to the event vertex is compared to the event start time, $t_{0}$. The difference between the two times gives the time correction needed. Fig. 33 shows an example of the time offset distribution for a thin and a thick tile.

The same procedure is used for the FT-Cal, however, all hits with energy greater than $10 \mathrm{MeV}$ are used with no requirement on the charge of the associated particle. The use of such a low energy threshold is important to be able to calibrate the crystals that are on the edges of the calorimeter. The measured time is then compared with the event start time, extracting both an overall offset and a charge-dependent correction, associated with a time-walk effect. The top-left panel of Fig. 34 shows the time offset as a function of the signal charge; this histogram profile is fit to a power law, $a / q^{\lambda}$, as shown in the topright panel to determine the time-walk correction. After applying this correction, the time offset distribution shown in the bottom plots of the same figure are fit to a simple Gaussian function to determine the global offset. The bottom right plot shows the final distribution with all corrections, showing a clear coincidence peak at 0 surrounded by the accidental peaks at multiples of $\pm 4.008 \mathrm{~ns}$ due to the RF beam structure. The time offset constant term is extracted for each crystal separately, while the time-walk constants are fit for all crystals together since no significant difference between the crystals was found. The resolution achieved with this procedure is reported in Section 8.
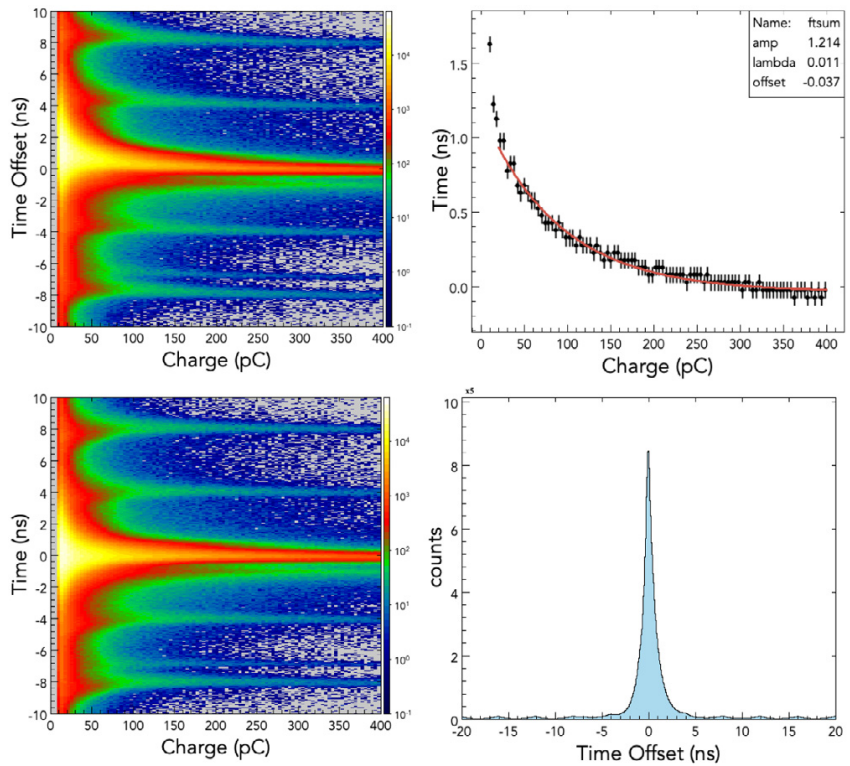

Fig. 34. Top: FT-Cal time offset dependence on the charge (left); the profile of the histogram is fit to a power law, $a / q^{\lambda}$. Bottom: FT-Cal time offsets after the time-walk correction and the subtraction of the residual constant term.

\section{Event reconstruction}

Reconstruction of the FT sub-detector information and the matching between the detectors to determine the type and three-momentum of the incident particles is implemented in the CLAS12 Java reconstruction framework. Details on the algorithms and implementation are provided in Ref. [19]. In the following we briefly summarize the main steps and final outputs.

FT-Cal hits are reconstructed from the analysis of the recorded FADC information to extract energy and time; hits are then associated based on position and time to form clusters whose energy and centroid position are used as an initial seed to define the three-momentum of the incident particles. Similarly, FT-Hodo hits are reconstructed from the FADC raw information and matched based on position and timing to form clusters of matching tiles in the two layers of the detector. These are matched to clusters in the calorimeter based on position and time to distinguish charged particles from neutrals. Finally, FT-Trk hits are also reconstructed from the raw data and geometrically grouped to form clusters in each of the detector layers separately. Combinations of clusters in the $x-y$ layers of each of the two sub-detectors are used to define crosses that are finally matched to calorimeter clusters to improve the determination of the impact point of the particle.

\section{Detector performance}

Data at different beam energies and with different trigger conditions have been analyzed to study and assess the FT performance. Results from the studies are detailed below.

\subsection{Acceptance}

The detector acceptance was studied in detail at the maximum beam energy the experiment operated at so far of $10.6 \mathrm{GeV}$. Data were recorded with a minimum-bias trigger based on the FT-Cal alone with a threshold on the measured cluster energy of $100 \mathrm{MeV}$. In the offline analysis, events were further selected, requiring a reconstructed electron via the matching of the FT-Cal cluster to FT-Hodo hits, and the associated FT-Cal cluster to have total energy greater than $500 \mathrm{MeV}$, seed energy greater than $300 \mathrm{MeV}$, and size greater than or equal to 4 

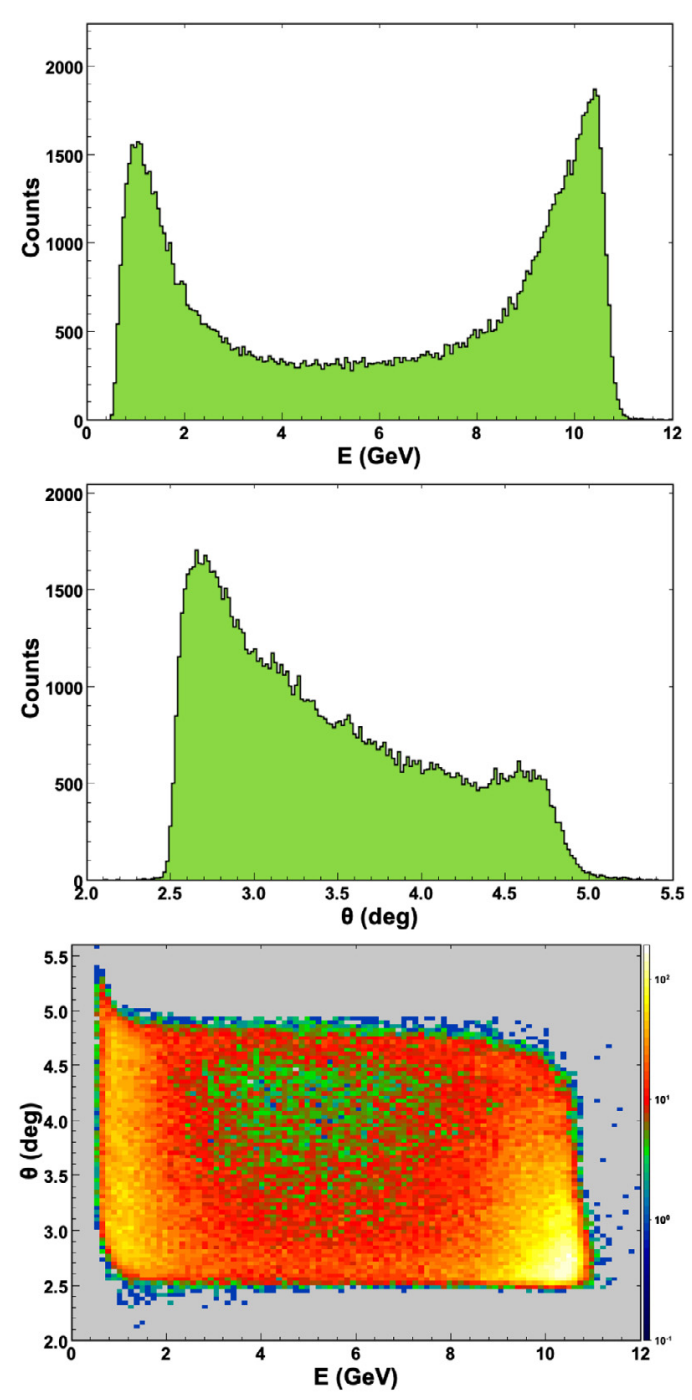

Fig. 35. FT acceptance for electrons as a function of energy (top), polar angle (middle), and of both variables (bottom) at $10.6 \mathrm{GeV}$ beam energy. The energy range goes from $500 \mathrm{MeV}$, as selected in the offline analysis, up to the end-point set by the beam energy where elastic scattering dominates. The $\theta$ range goes from the minimum angle of $2.5^{\circ}$ to $\sim 5^{\circ}$. The two-dimensional distribution shows the effect of the CLAS12 solenoid field on low-momentum electrons that start from $\theta \sim 2^{\circ}$ and are bent into the detector acceptance.

crystals. The resulting event distributions as a function of the electron energy and polar angle are shown in Fig. 35.

The energy coverage extends from $500 \mathrm{MeV}$, as selected in the offline analysis, up to the end-point set by the beam energy where elastic scattering dominates. Close to the energy end-point, the detector resolution is expected to worsen significantly because of saturation of the FT-Cal preamplifiers and FADCs that are optimized for the design energy range of $0.5-4.5 \mathrm{GeV}$. The $\theta$ range extends from the minimum angle of $2.5^{\circ}$ to $\sim 5^{\circ}$. The two-dimensional distribution shows the effect of the CLAS12 solenoid field on low-momentum electrons starting from $\theta \sim 2^{\circ}$ that are bent into the detector acceptance. The detector acceptance matches and partially exceeds the design specifications.

\subsection{Energy resolution and electromagnetic shower reconstruction}

Within the detector acceptance, the energy resolution was studied based on elastic scattering and $\pi^{0}$ decay to two photons, as discussed in Section 6. The results indicate the currently achieved resolution is larger than the design value by about $1 \%$ at $2 \mathrm{GeV}$. The reasons

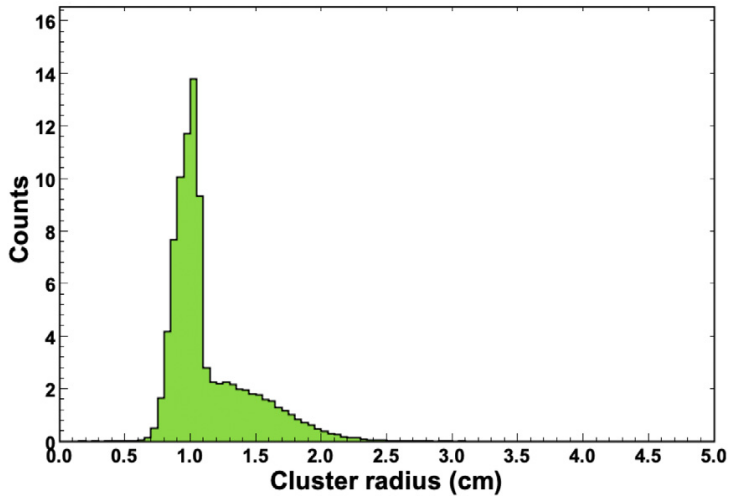

Fig. 36. Radius of the FT-Cal shower for charged particles. A clear peak at $\sim 1 \mathrm{~cm}$ associated with electron-induced electromagnetic showers overlaps with a broader distribution due to hadronic showers.

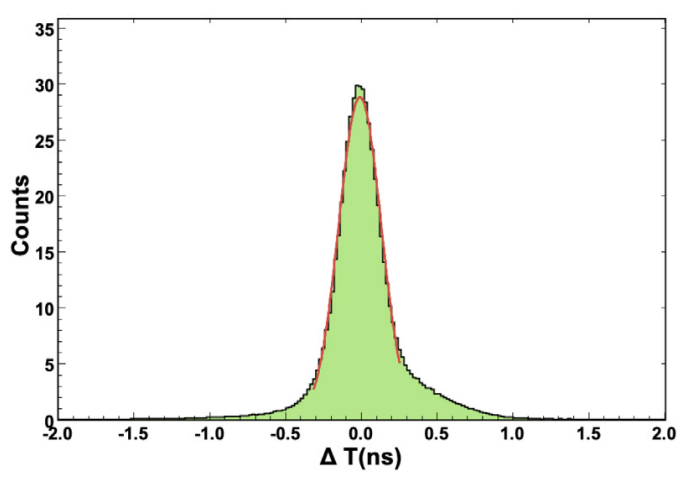

Fig. 37. Time resolution for electrons detected in the FT with energy greater than $500 \mathrm{MeV}$, seed energy greater than $300 \mathrm{MeV}$, and cluster size greater than or equal to 4. The histogram shows the time difference between the FT time projected back to the event vertex and the RF signal time. The Gaussian fit gives a resolution $\sigma \sim 140$ ps.

for this discrepancy can be multi-fold. First, the energy calibration of individual crystals has shown a significant spread in the energyto-charge conversion that was not foreseen in the initial estimates. This spread, likely due to the non-uniformity of the crystal light yield, can contribute to a worsening of the resolution because it results in a non-homogeneous detector response. Second, as a consequence of the crystal non-uniformity, the threshold applied in the cluster reconstruction is for some crystals larger than the $10 \mathrm{MeV}$ used in the simulation studies and prototype analyses.

The shower profile in the FT-Cal was studied and compared to Monte Carlo simulations for different particle species. Fig. 36 shows the shower radius, defined as the square root of the second moment of the shower, for charged particles, i.e. particles associated with a cluster in the calorimeter with matching hits in the hodoscope. A clear peak with radius of $\sim 1 \mathrm{~cm}$ associated with electrons is clearly visible, overlapping a broader distribution associated with hadronic showers. The shower profile and, specifically the cluster radius, can therefore be used to discriminate between different particle types.

\subsection{Timing resolution}

The timing resolution for electrons and photons was evaluated from beam data by correlating the reconstructed cluster time from the FT-Cal to either the RF signal that is synchronous with the CEBAF accelerator beam bunches or the event start time derived from the CLAS12 FTOF system [23]. Specifically, the electron time resolution was studied correlating the FT time projected back to the event vertex to the RF signal time. The difference of these two times for $10.6 \mathrm{GeV}$ data is 


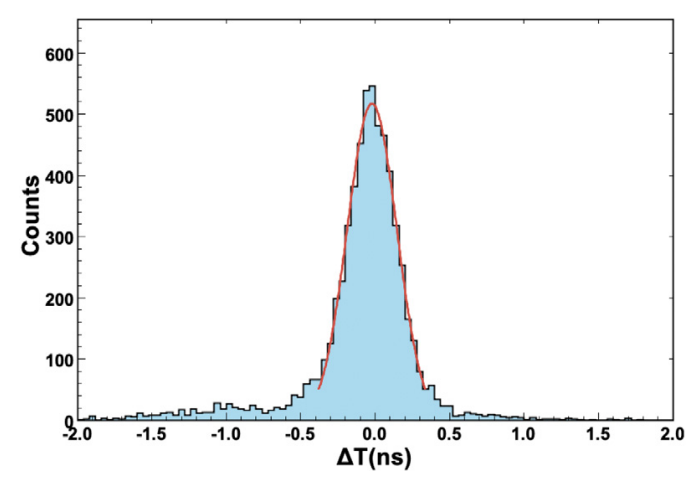

Fig. 38. Time resolution for photons detected in the FT with energy greater than $500 \mathrm{MeV}$, seed energy greater than $300 \mathrm{MeV}$, and cluster size greater than or equal to 4 . The histogram shows the time difference between the FT time projected back to the event vertex and the event start time derived from the CLAS12 FTOF detector for events where an electron is identified in the CLAS12 Forward Detector. The Gaussian fit gives a resolution $\sigma \sim 150$ ps.

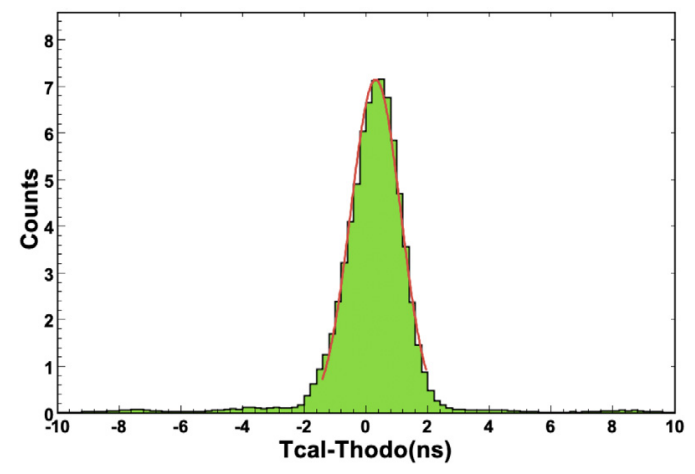

Fig. 39. Time difference between the calorimeter and hodoscope clusters for reconstructed electrons. The Gaussian fit to the distribution gives $\sigma \sim 0.8 \mathrm{~ns}$.

shown in Fig. 37 for electrons with energy greater than $500 \mathrm{MeV}$, cluster seed energy greater than $300 \mathrm{MeV}$, and cluster size greater than or equal to 4 crystals: a Gaussian fit to the distribution gives $\sigma \sim 140$ ps. The tails of the distribution are due to low-energy clusters close to the applied selection threshold, and are expected to be reduced by improvements of the time-walk correction that are currently under study.

While this estimate of the time resolution relies solely on the FT reconstruction, an alternative measure can be performed by selecting photons detected in the FT and correlating their time to the event start time determined from other particles detected in CLAS12. This analysis was performed for events with an electron detected in the CLAS12 Forward Detector whose start time is determined based on the FTOF system and a photon detected in the FT with energy greater than $500 \mathrm{MeV}$, cluster seed energy greater than $300 \mathrm{MeV}$, and cluster size greater than or equal to 4 crystals. The photon FT time projected back to the event vertex was correlated with the event start time as shown in Fig. 38. A Gaussian fit to the distribution gives $\sigma \sim 150 \mathrm{ps}$, slightly larger but consistent with the electron timing resolution.

While the FT hit time is determined by the calorimeter since this is the component with the best timing resolution, the time correlation between the individual FT detectors is important to match the signals detected in the three sub-components and minimize accidentals. Fig. 39 shows the time difference of the reconstructed calorimeter and hodoscope clusters for detected electrons with $\sigma \sim 0.8 \mathrm{~ns}$, dominated by the hodoscope resolution. The value is consistent with the design resolution for the hodoscope of $<1 \mathrm{~ns}$.

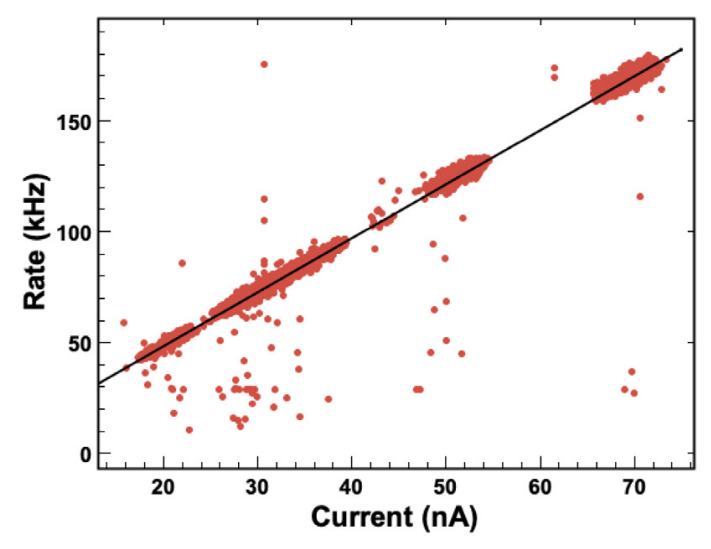

Fig. 40. FT trigger rate as a function of the beam current. The measurements are consistent with a linear dependence up to the maximum CLAS12 luminosity of $10^{35} \mathrm{~cm}^{-2} \mathrm{~s}^{-1}$, which is obtained at a current of $75 \mathrm{nA}$ on a 5-cm-long liquid-hydrogen target. The points that deviate from the linear slope correspond to measurements with unstable beam conditions.

\subsection{Trigger performance}

The FT is used as an active component of the CLAS12 trigger system to identify events in which electrons or photons are detected in the system. This is achieved by reconstructing in real time clusters in the calorimeter with or without geometrical and time matching with hodoscope tiles. Details on the trigger algorithms, their implementation, and validation are provided in Ref. [20], while here we focus only on reporting the performance in terms of linearity of the trigger rate as a function of luminosity. This was studied performing a luminosity scan and recording the FT trigger rate at the input of the data acquisition system. Fig. 40 shows the measured dependence. These results confirm the linearity of the FT trigger up to the maximum luminosity foreseen for the experiment.

\section{Conclusions}

This paper describes the layout and performance of the CLAS12 Forward Tagger. This system was designed to detect electrons scattered at very small angles, $2.5^{\circ}$ to $4.5^{\circ}$, and to perform measurements of hadronic reactions in the kinematics of quasi-real photoproduction. In this regime, the virtual photon exchanged by the electron interaction with the target has very low four-momentum transfer $Q^{2}$ and can be considered as a real photon. These kinematics are ideally suited for the study of hadron production and spectroscopy, extending the physics reach of the CLAS12 experiment beyond its original scope.

The Forward Tagger, composed of an electromagnetic calorimeter for electron detection and energy measurements, a hodoscope to distinguish electrons from photons, and a tracker to precisely measure the electron scattering plane, was designed to be permanently installed in CLAS12 as an integral part of the beamline. After extensive simulation and detector prototyping studies, the three Forward Tagger detectors were assembled and tested separately prior to integration and installation in CLAS12. Upon installation, the full system was commissioned first with cosmic ray data taking and then with beam during the CLAS12 engineering run. These studies enabled us to optimize the detector configuration and to consolidate the calibration procedures for all system components before the start of physics experiments with CLAS12.

The system response has been studied based on different physics reactions to determine acceptance, energy and timing resolution, and trigger performance. While further improvements are expected based on refinements of the calibration procedures and reconstruction algorithms, the Forward Tagger performance is qualitatively in agreement with the system design specifications, enabling the physics program for which this detector system was designed. 


\section{Acknowledgments}

The authors would like to thank the engineering and technical staff of Jefferson Lab, the Italian Istituto Nazionale di Fisica Nucleare, the University of Edinburgh, and the University of Glasgow, for their effort and support during the design, construction, and operation of the Forward Tagger. Special thanks to Gianni Nobili, Andrea Rottura, and Diego Torazza for their assistance in this project. Many achievements in the development of the Forward Tagger system would have not beem possible without the support of Nathan Baltzell, Sergey Boyarinov, Chris Cuevas, Gagik Gavalian, Ben Raydo, Maurizio Ungaro, and Veronique Ziegler. This work was supported in part by the Italian Istituto Nazionale di Fisica Nucleare, the Scottish Universities Physics Alliance (SUPA), United Kingdom, the United Kingdom's Science and Technology Facilities Council, the French Commissariat à l'Energie Atomique, the U.S. Department of Energy, Office of Science, USA, Office of Nuclear Physics under contract DE-AC05-06OR23177, and the National Science Foundation, USA under grant NSF PHY-1229373.

\section{References}

[1] M. Battaglieri, et al., E12-11-005, Meson spectroscopy with low $Q^{2}$ electron scattering in CLAS12, a CLAS collaboration proposal to PAC37, 2011.

[2] V.D. Burkert, et al., The CLAS12 spectrometer at Jefferson laboratory, Nucl. Instrum. Methods A (2020) (See this issue) (in press).

[3] S. Boyarinov, et al., The CLAS12 data acquisition system, Nucl. Instrum. Methods A (2020) (See this issue) (in press).

[4] Y.G. Sharabian, et al., The CLAS12 high threshold Cerenkov counter, Nucl. Instrum. Methods A (2020) (See this issue) (in press).

[5] R. Fair, et al., The CLAS12 superconducting magnets, Nucl. Instrum. Methods A (2020) (See this issue) (in press).

[6] M.D. Mestayer, et al., The CLAS12 drift chamber system, Nucl. Instrum. Methods A (2020) (See this issue) (in press).

[7] CMS Collaboration, The Electromagnetic Calorimeter Project: Technical Design Report, CERN-LHCC-97-33, 1997.
[8] F.X. Girod, et al., Measurement of deeply virtual compton scattering beam-spin asymmetries, Phys. Rev. Lett. 100 (2008) 162002.

[9] PANDA Collaboration, Technical design report for the PANDA forward spectrometer calorimeter, 2017, arXiv:1704.02713.

[10] T. Horn, et al., Scintillating crystals for the Neutral Particle Spectrometer in Hall C at JLab, 2019, arXiv:1911.11577.

[11] E. Auffray, et al., Performance of ACCOS, an automatic crystal quality control system for the PWO crystals of the CMS calorimeter, Nucl. Instrum. Methods A 456 (2001) 325-341.

[12] S. Fegan, et al., Assessing the performance under ionising radiation of lead tungstate scintillators for EM calorimetry in the CLAS12 Forward Tagger, Nucl. Instrum. Methods A 789 (2001) 101-108.

[13] A. Celentano, et al., Design and realization of a facility for the characterization of Silicon Avalanche PhotoDiodes, JINST 9 (2014) T09002.

[14] M. Battaglieri, et al., CLAS12 forward tagger (FT) technical design report, 2012, http://www.ge.infn.it/ batta/jlab/ft-tdr.2.0.pdf.

[15] A. Acker, et al., The CLAS12 Micromegas vertex tracker, Nucl. Instrum. Methods A (2020) (See this issue) (in press).

[16] S. Stepanyan, et al., The CLAS12 beamline and its performance, Nucl. Instrum. Methods A (2020) (See this issue) (in press).

[17] M. Ungaro, et al., The CLAS12 Geant4 simulation, Nucl. Instrum. Methods A (2020) (See this issue) (in press).

[18] G.M. B. Buonomo, P. Valente, Performance and upgrade of the DAFNE beam test facility (BTF), IEEE Trans. Nucl. Sci. 52 (2005) 824.

[19] V. Ziegler, et al., The CLAS12 software framework and event reconstruction, Nucl. Instrum. Methods A (2020) (See this issue) (in press).

[20] S. Boyarinov, et al., The CLAS12 trigger system, Nucl. Instrum. Methods A (2020) (See this issue) (in press).

[21] E. Fanchini, et al., FT calorimeter response to cosmic ray data during the precommissioning phase, 2016, CLAS12- Note 2016-005. https://misportal.jlab.org/ mis/physics/clas12/viewFile.cfm/2016-005.pdf?documentId=31.

[22] E. Fanchini, et al., FT calorimeter response to cosmic ray during the commissioning phase at JLab with high and low gain preamplifiers, 2017, CLAS12-Note 2017-006. https://misportal.jlab.org/mis/physics/clas12/viewFile. $\mathrm{cfm} / 2017-006 . p d f$ ?documentId=41.

[23] D.S. Carman, et al., The CLAS12 forward time-of-flight system, Nucl. Instrum. Methods A (2020) (See this issue) (in press). 